\title{
Circuit
}

Musiques contemporaines

\section{Extraits de partitions en création en 2009-2010}

Analia Llugdar, Sylvain Pohu, Robert Aitken, Wolf Edwards, Vincent Ho, Cléo Palacio-Quintin, Eldritch Priest, Cassandra Miller, Gabriel Dharmoo, Gabriel Dufour-Laperrière, Snezan Nesic, Nicolas Bernier, André Ristic, Gilles Tremblay, René Orea-Sanchez, Brian Current, Michel Gonneville, Dániel Péter Biró, Simon Bertrand, Éric Champagne, Frédéric Chiasson, Benoît Côté, Maxime Goulet, Marielle Groven et Pierre Olivier Roy

Volume 20, numéro 1-2, 2010

La musique contemporaine d'hier à demain

URI : https://id.erudit.org/iderudit/039643ar

DOI : https://doi.org/10.7202/039643ar

Aller au sommaire du numéro

Éditeur(s)

Les Presses de l'Université de Montréal

ISSN

1183-1693 (imprimé)

1488-9692 (numérique)

Découvrir la revue

Citer ce document

Llugdar, A., Pohu, S., Aitken, R., Edwards, W., Ho, V., Palacio-Quintin, C., Priest, E., Miller, C., Dharmoo, G., Dufour-Laperrière, G., Nesic, S., Bernier, N., Ristic, A., Tremblay, G., Orea-Sanchez, R., Current, B., Gonneville, M., Biró, D. P., Bertrand, S., Champagne, É., Chiasson, F., Côté, B., Goulet, M., Groven, M. \& Roy, P. O. (2010). Extraits de partitions en création en 2009-2010. Circuit, 20(1-2), 54-90. https://doi.org/10.7202/039643ar 

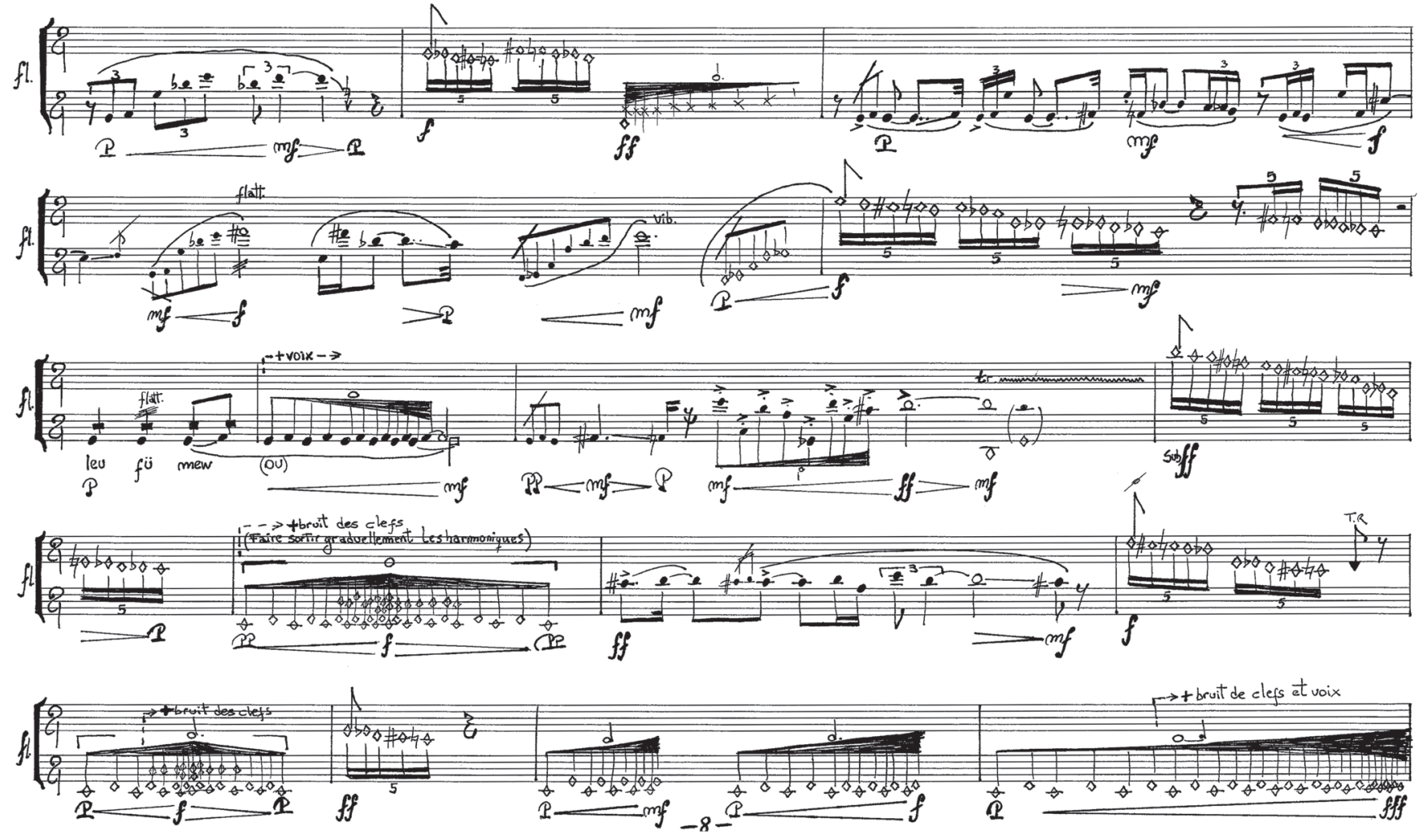

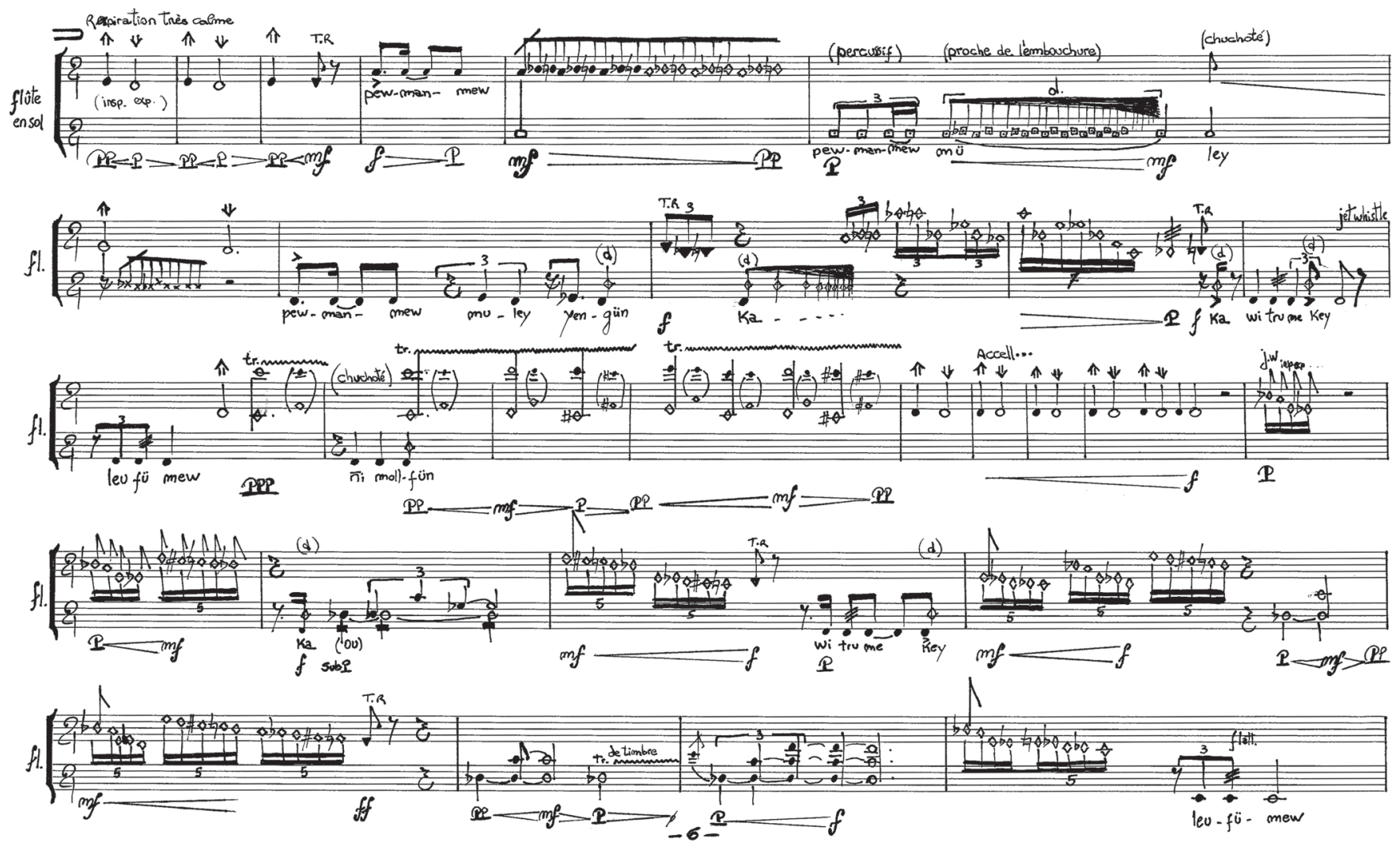

Pour flûte en do, flûte alto et électroniques. À Marie-Hélène Breault.

Création: 19 mars 2010, Chapelle historique du Bon-Pasteur, Montréal.

Ensemble: Erreur de type 27 


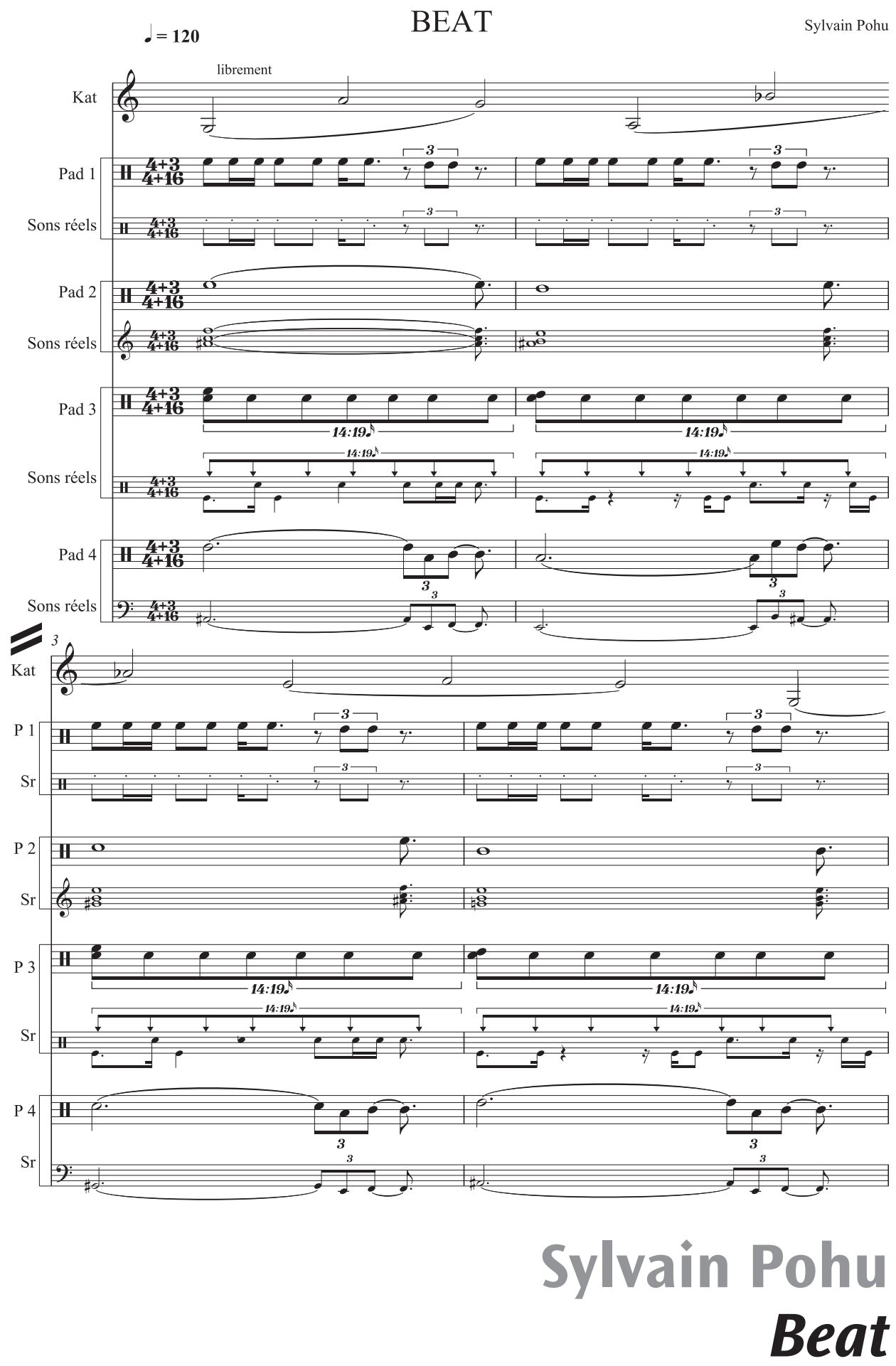




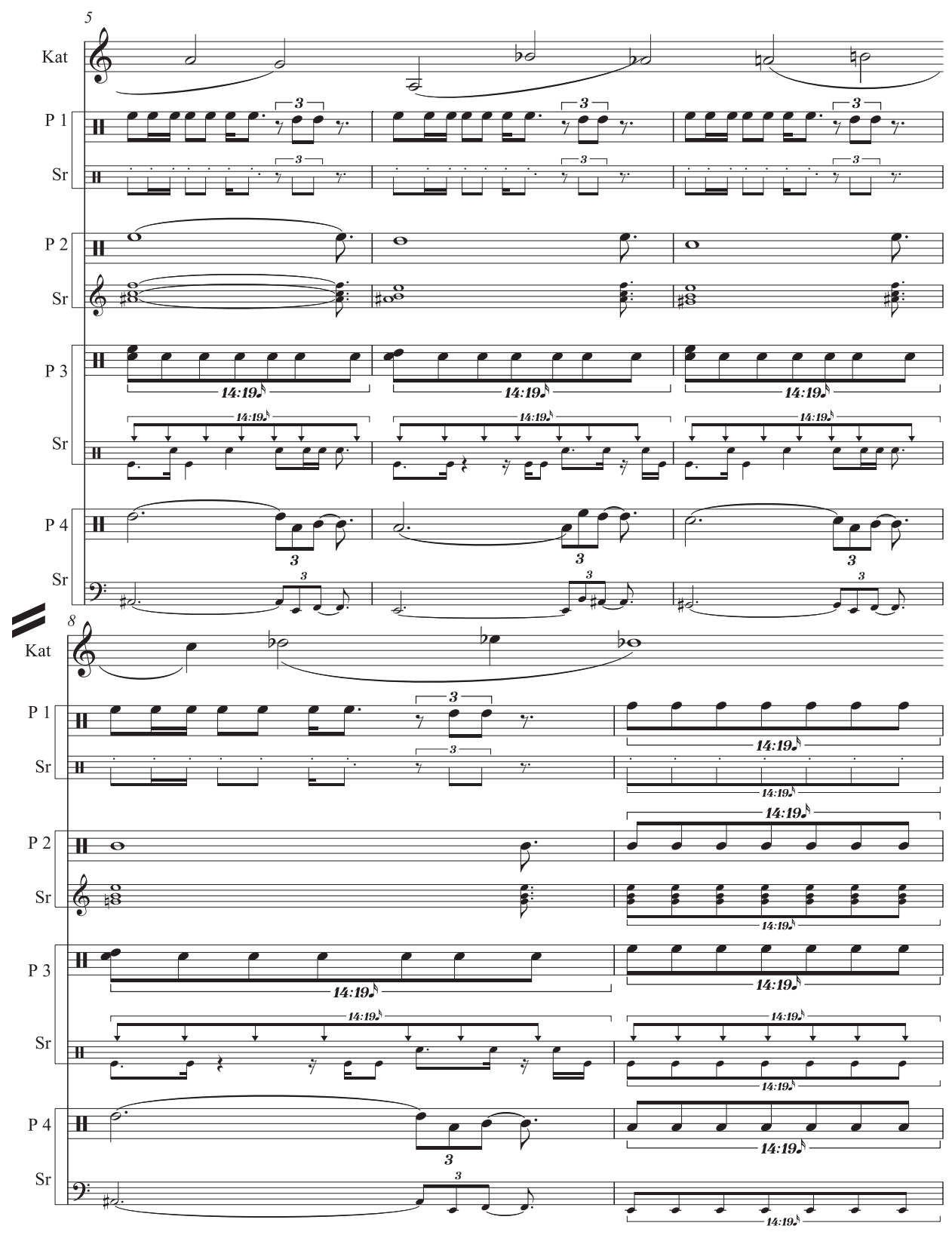

Vidéo-musique.

Création : 7 mai 2010, Usine C, Montréal (Festival Elektra de l'ACREQ).

Ensemble: Sixtrum 
(1020) (3) (12

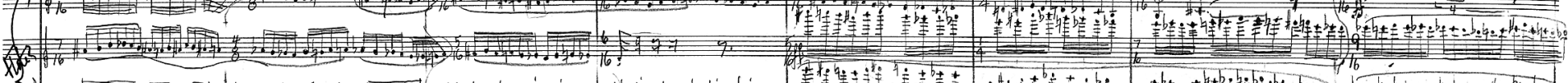
3)

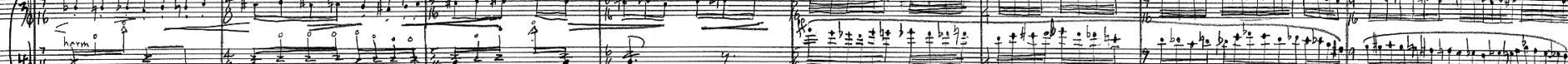

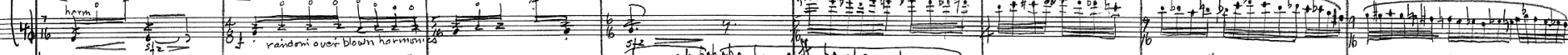

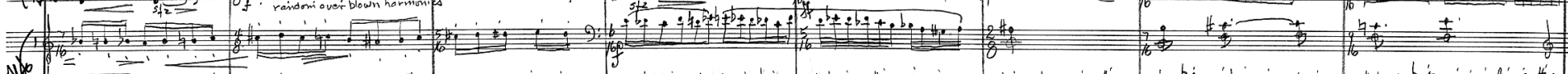

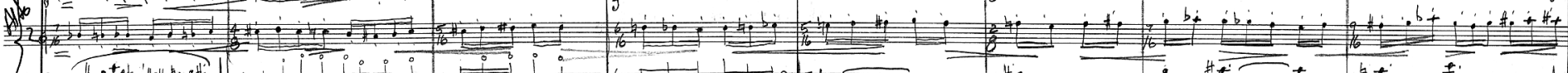
37. (1) (2) 10. 1. (2) 4

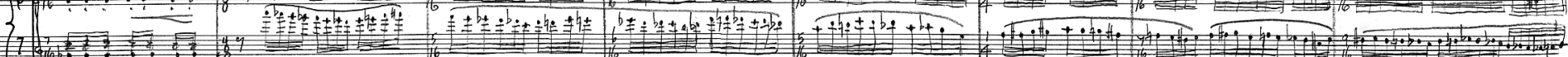
W W W W W 3 (3) 1. 3 . ?

(1)

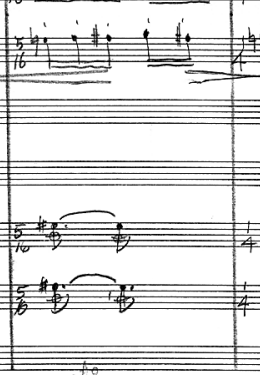

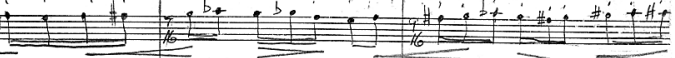




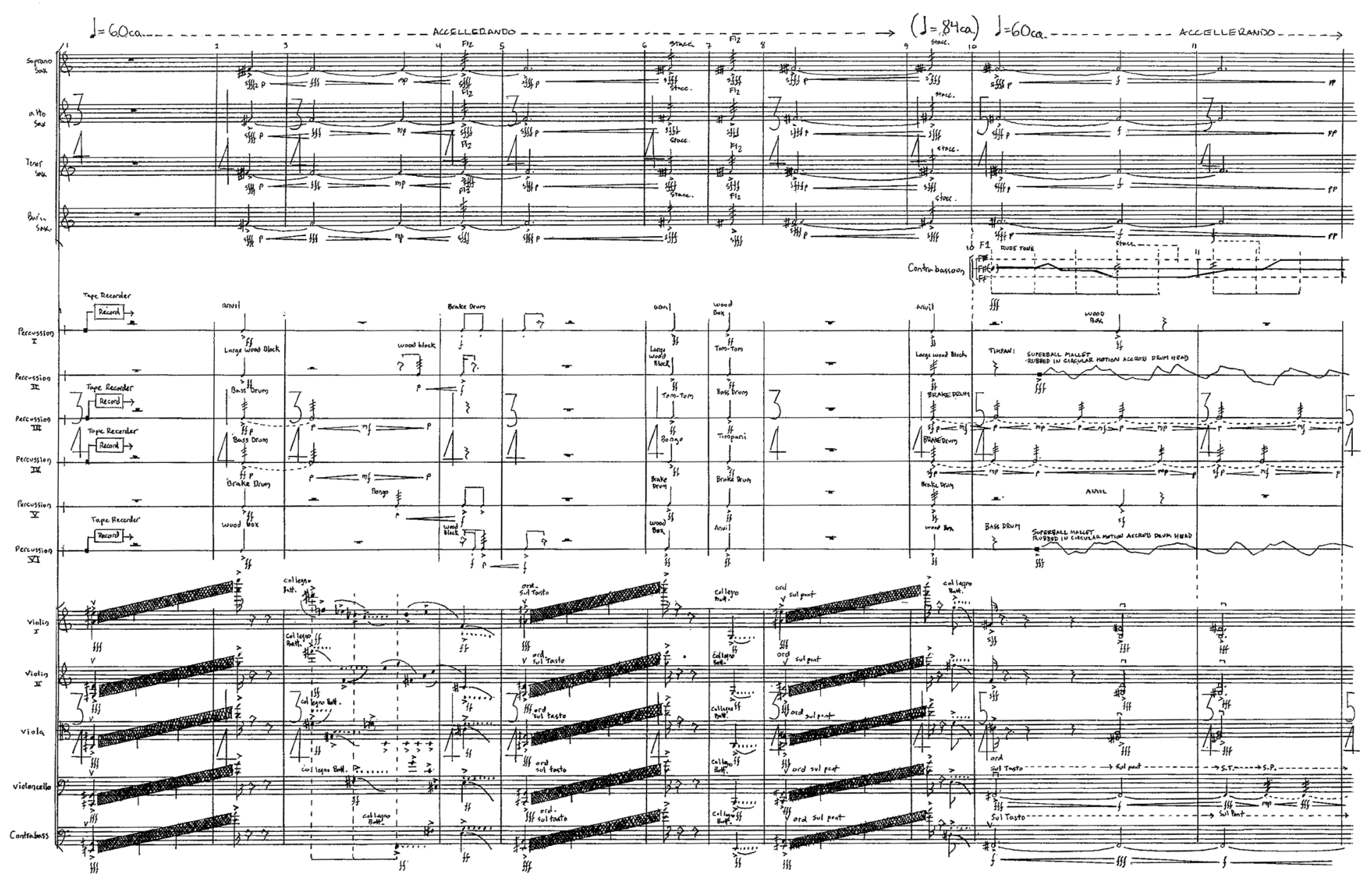

\section{Wolf Edwards}




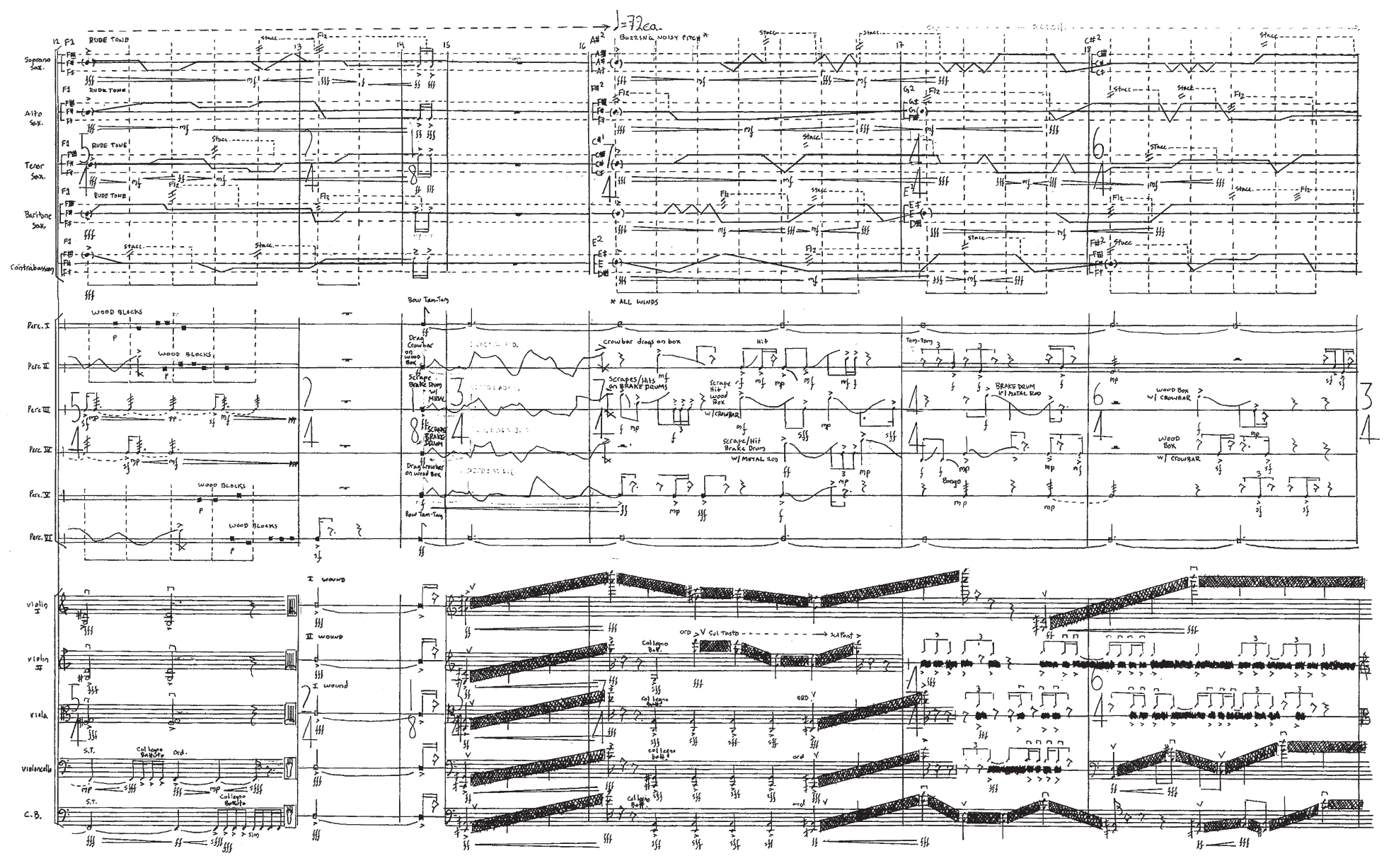

Création : 28 octobre 2010, Salle Pierre-Mercure, Montréal. 


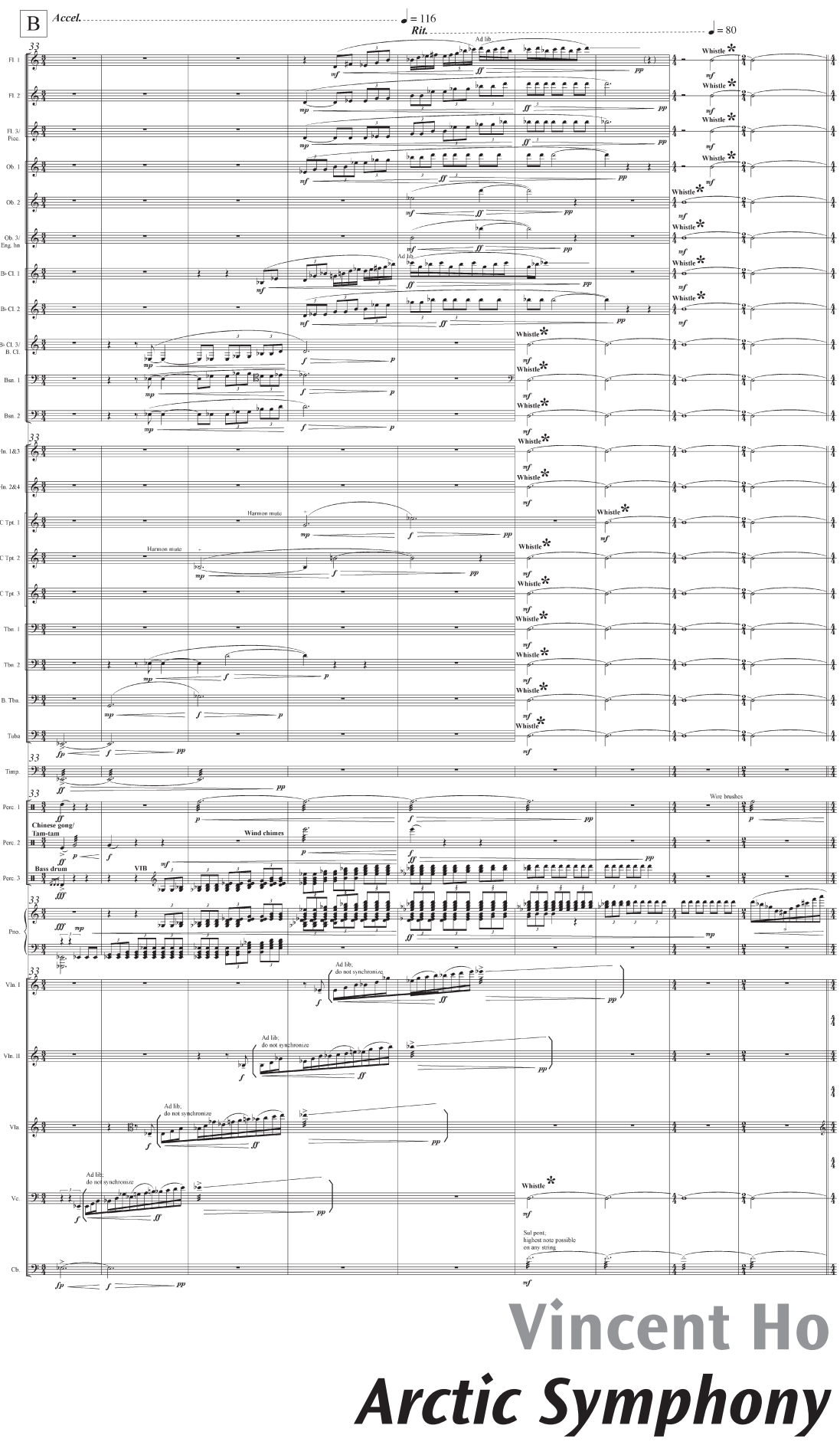




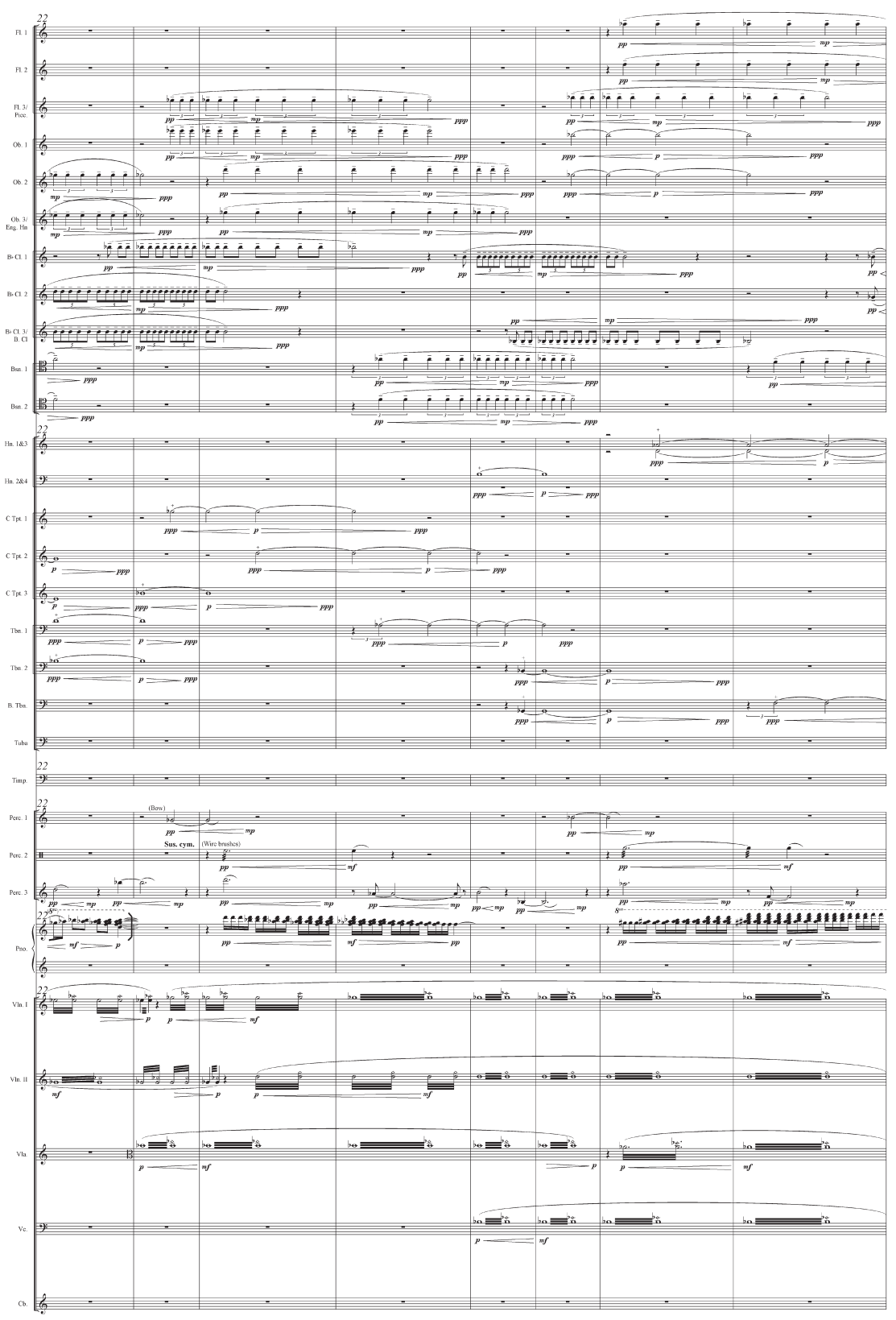

Création: 6 février 2010, Centennial Concert Hall, Winnipeg (WSO's New Music Festival). Ensemble: Winnipeg Symphony Orchestra 

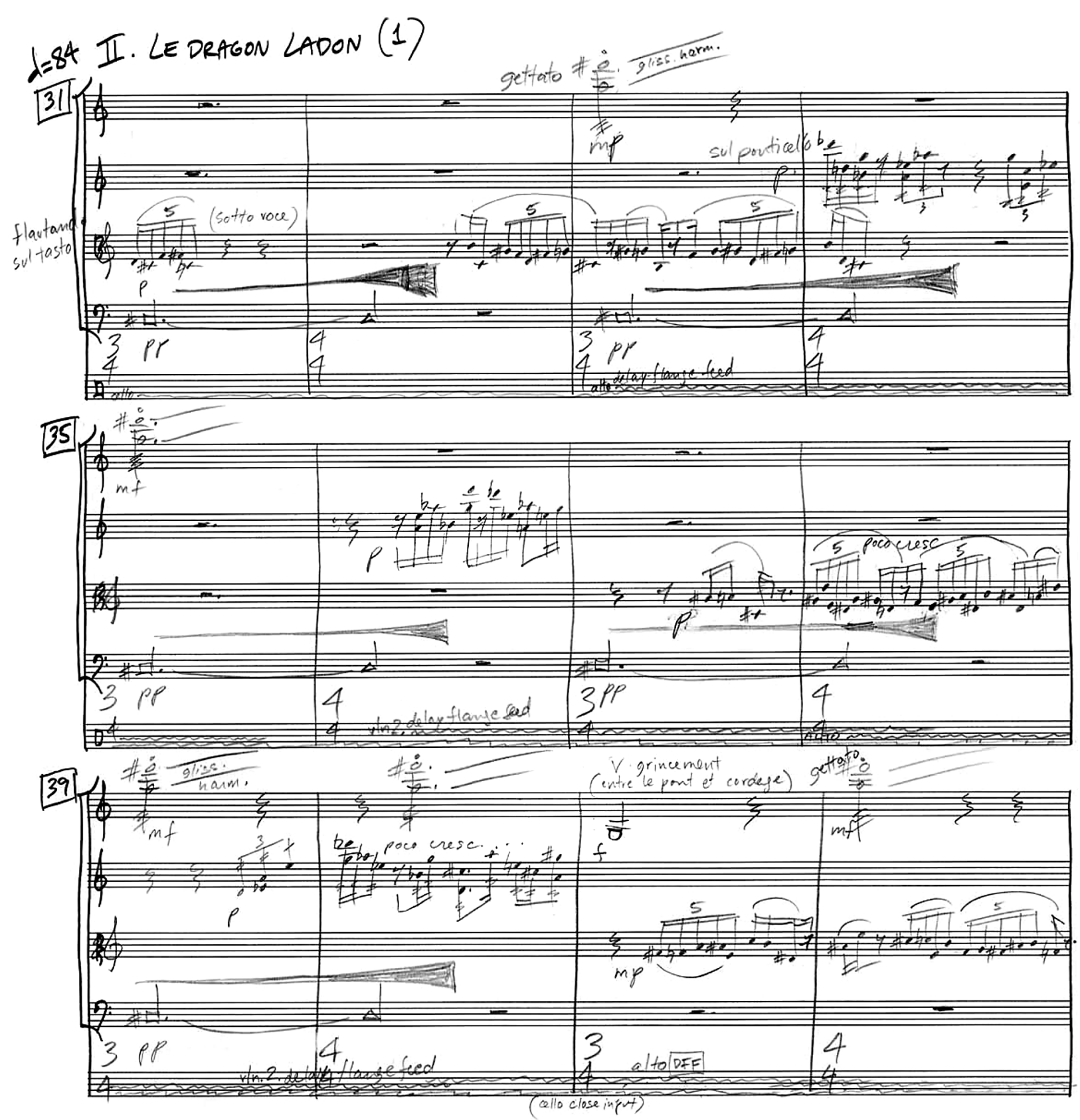

\section{Cléo Palacio-Quintin Le jardin des Hespérides}



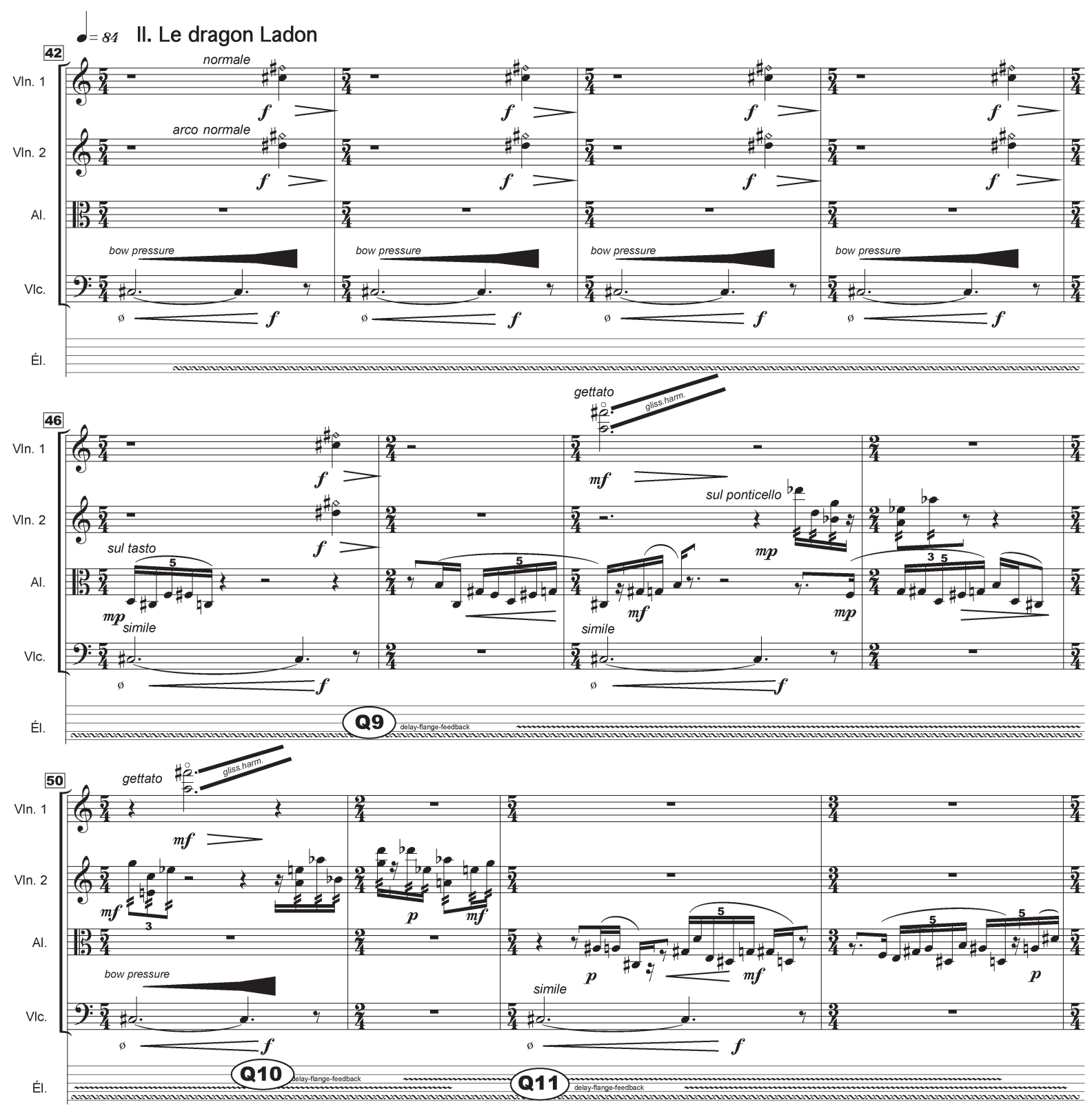

Pour quatuor à cordes.

Création: 23 avril 2010, Chapelle historique du Bon-Pasteur, Montréal,

Salon des compositeurs 3.

Ensemble: Quatuor Bozzini 
Thesaurus of Omnibook

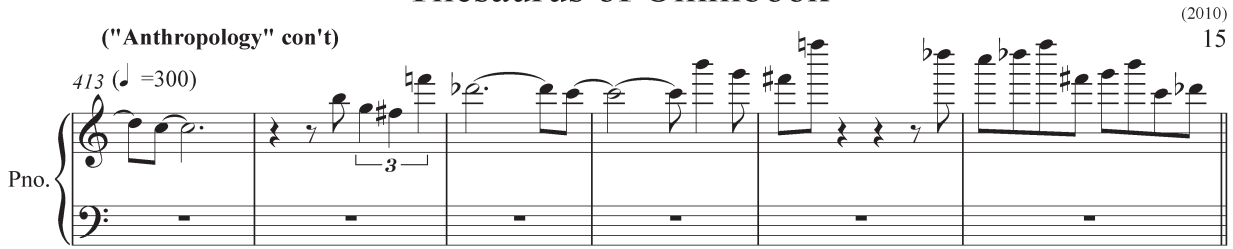

("Ultrapolation of Two Notes" con't)
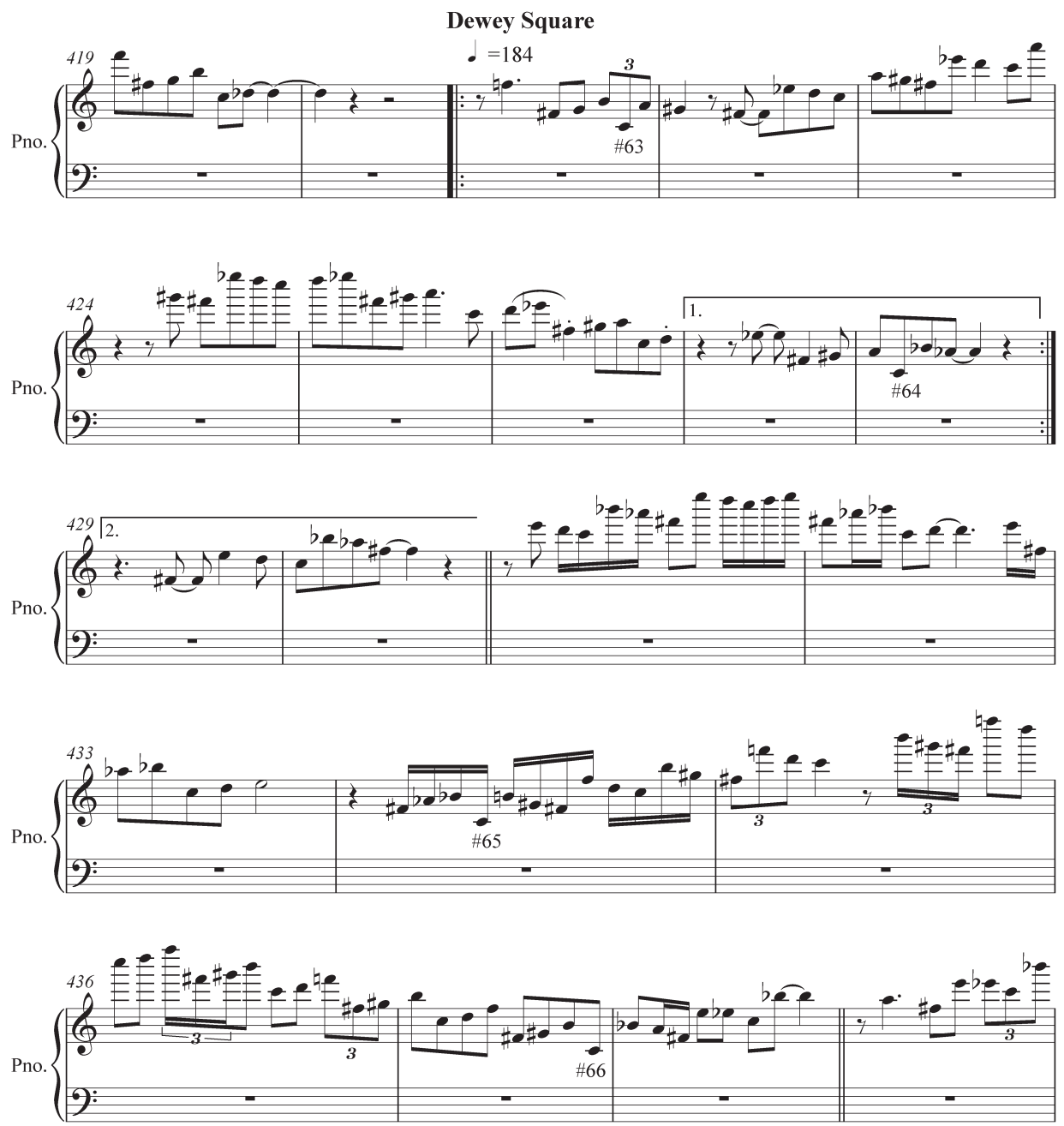

\section{Eldritch Priest Thesaurus of Omnibook}




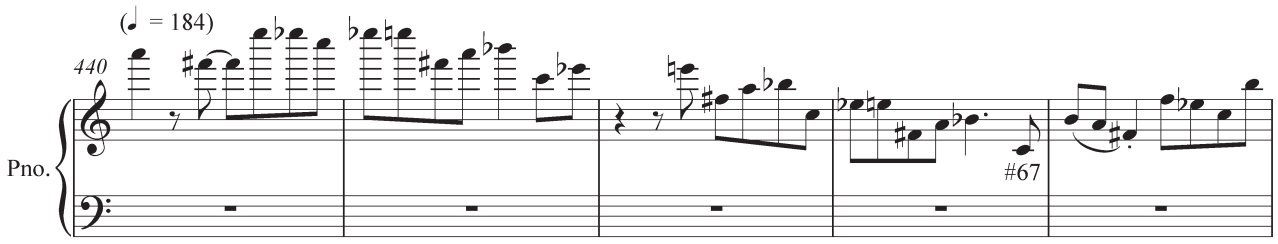

("Ultrapolation of Two Notes" con't)
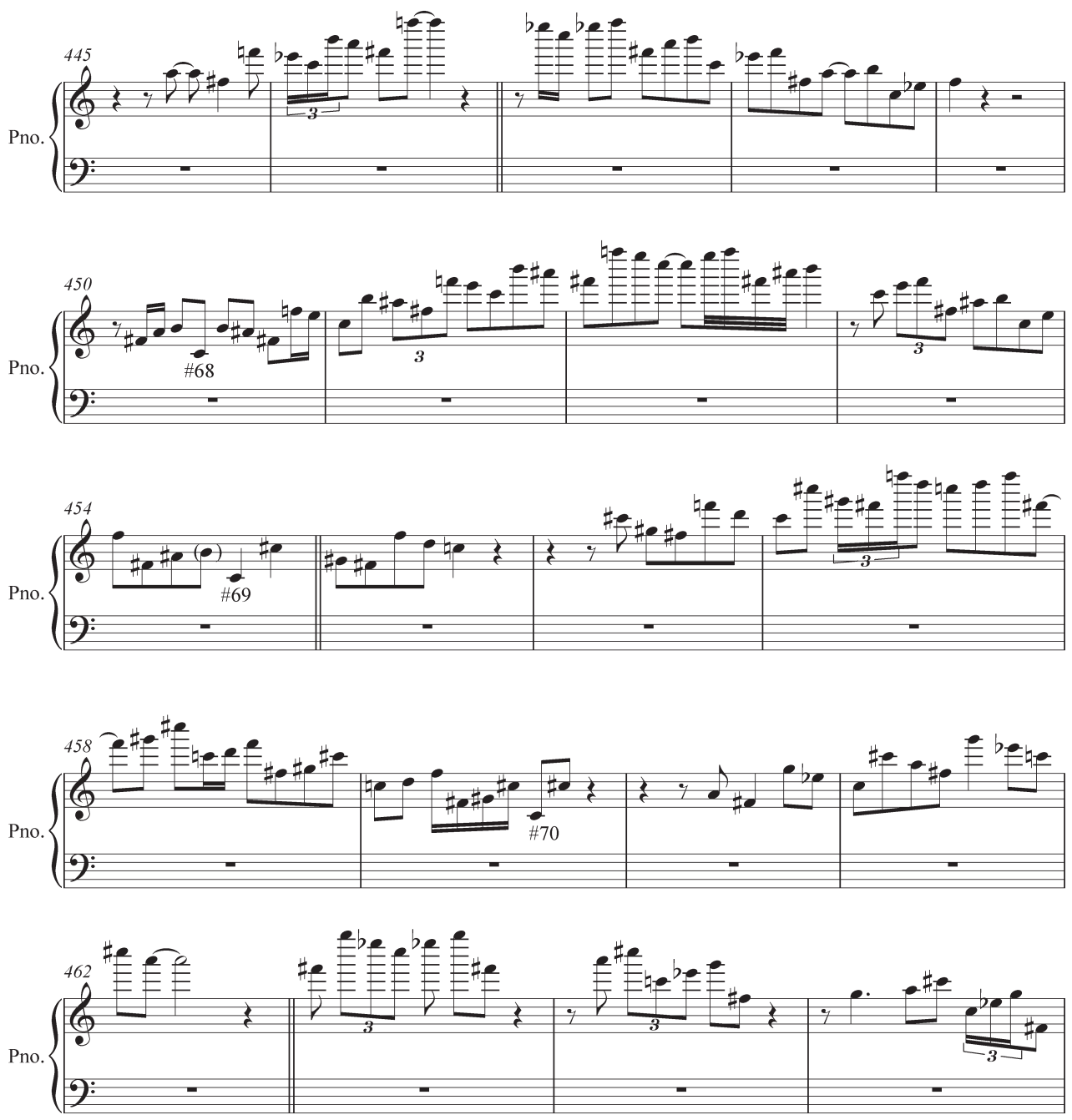

Création: neither/nor festival (House of Cards), automne 2010, Toronto. 


\section{Bel Cinto}

Cassandra Miller

Ensemble A: alto flute, bb clarinet, guitar, mezzo-soprano

Ensemble B: violin, viola, cello

Ensembles A and B read from two separate scores (score A and B) and proceed alongside each other without relation, for the duration of the piece ( 20 minutes), as if two pieces played simultaneously. Ensemble B begins a couple of seconds after Ensemble A, and the piece is finished when both groups are independently finished.

Bel Canto - Score A

Ensemble A: Alto Flute, (Bb) Clarinet, Acoustic Guttar, Mezzo-soprano
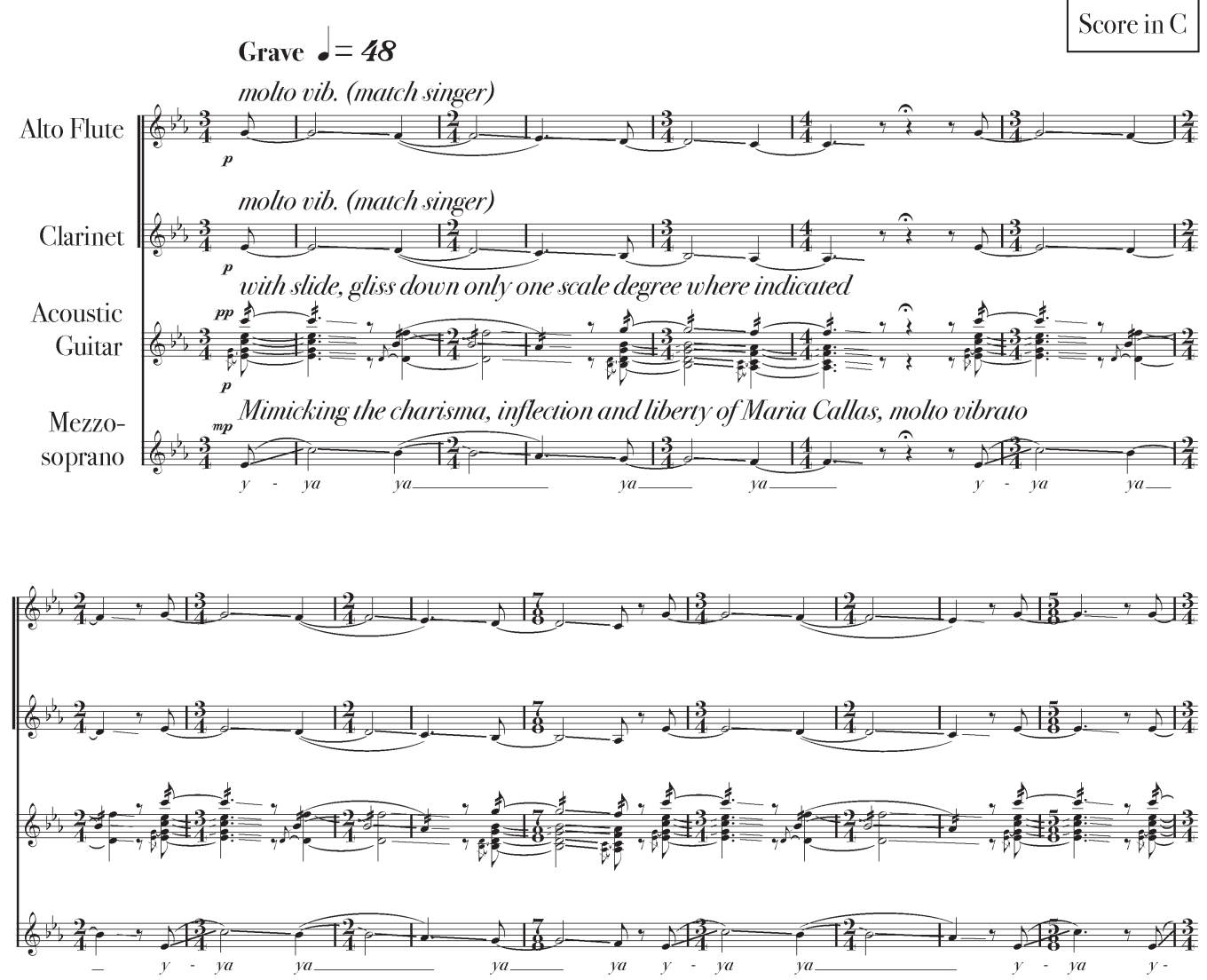


\section{Bel Canto - Score B \\ Ensemble B: Violin, Viola, Cello}

Vibrato notation $-\overline{\mathrm{m} 3}$

This sign above the statt indıcates width of vibrato. The solid upper line represents the pitch as indicated on the staff, and the lower dashed line indicates the distance below this pitch reach by vibrato. M3 = major third, $\mathrm{m} 2$ = minor second, etc.
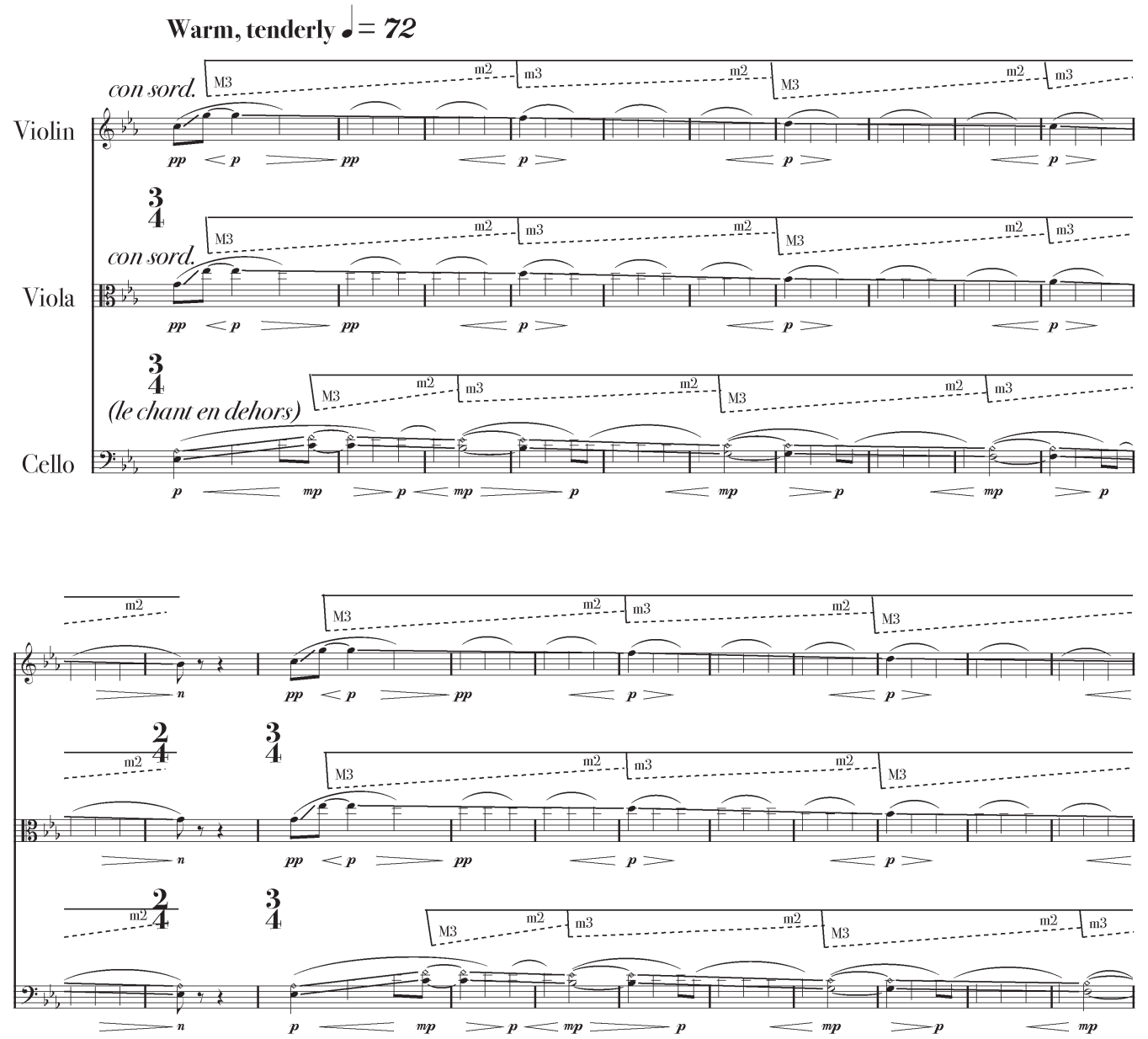

Création : 14 mai 2010, Chapelle historique du Bon-Pasteur, Montréal. 


\section{Vaai Irandu}

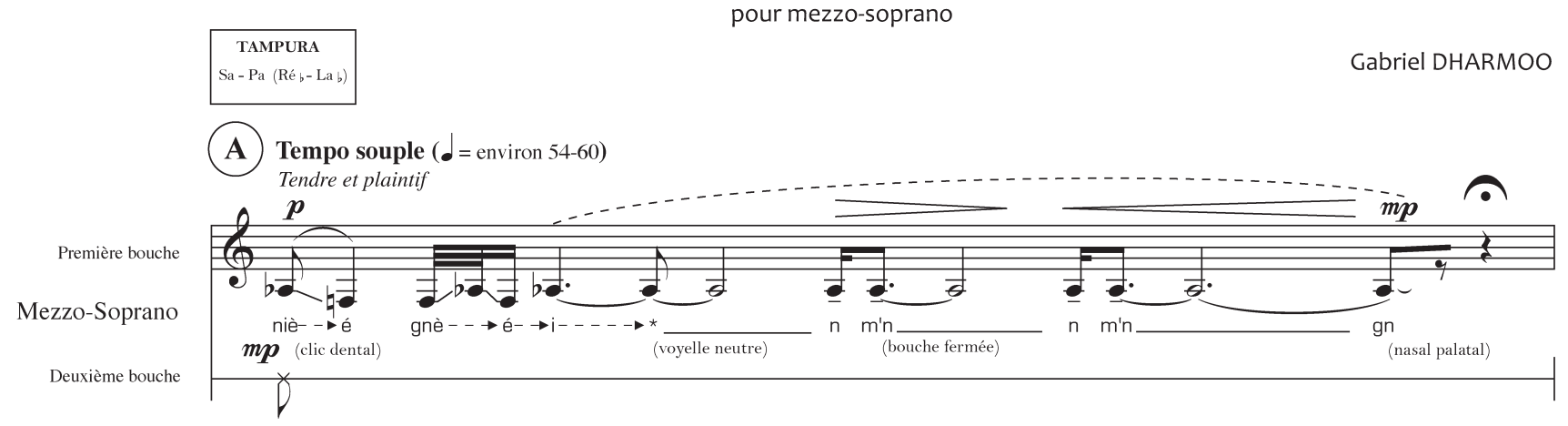

M. S.
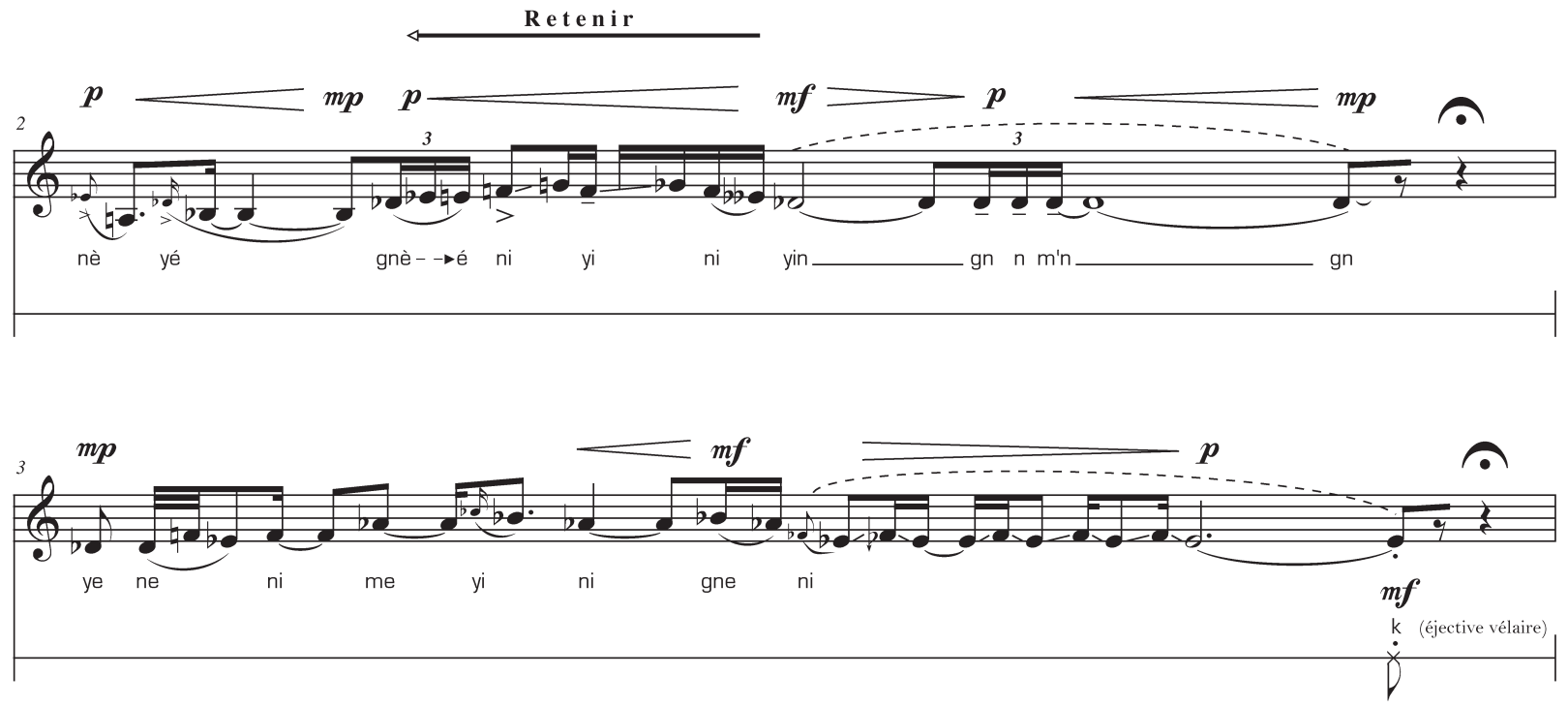

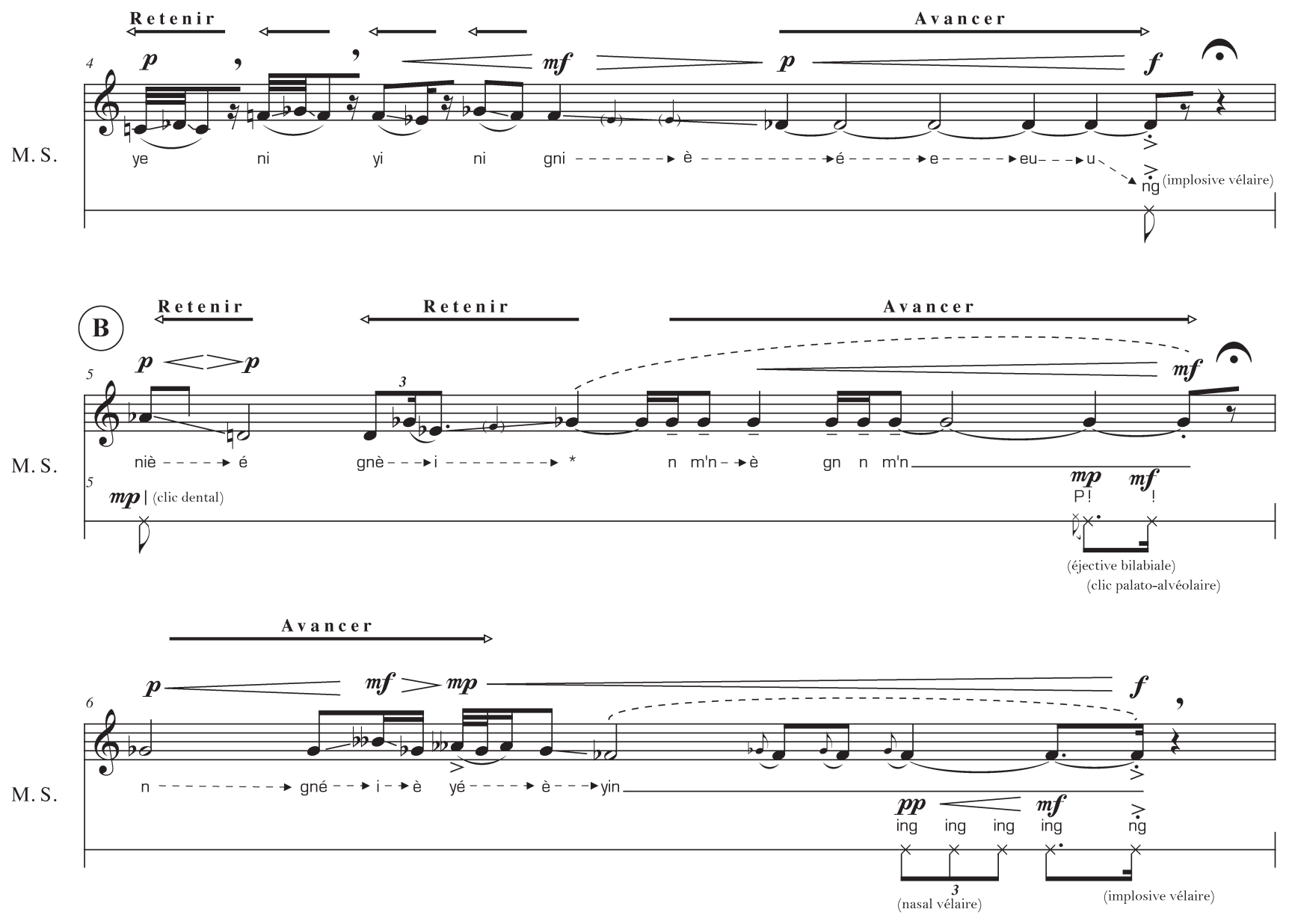

2

Création: 3 mars 2010, eXcentris, salle Cassavetes, Montréal.

Ensembles: Ensemble contemporain de Montréal (ECM+) et Proxima Centauri (France)/ Véronique Lacroix. 

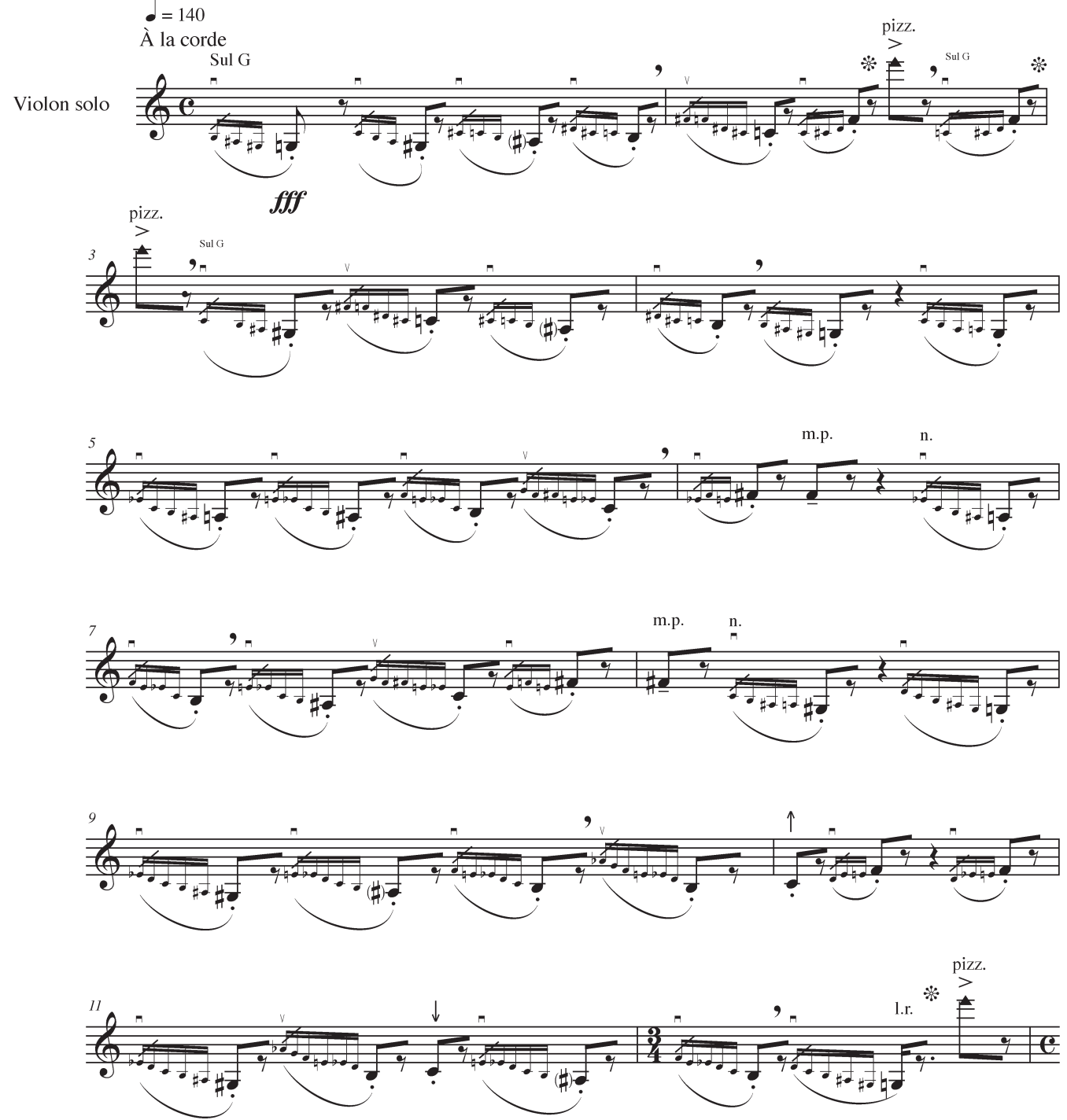

\section{Gabriel Dufour-Laperrière} De l'intérieur 

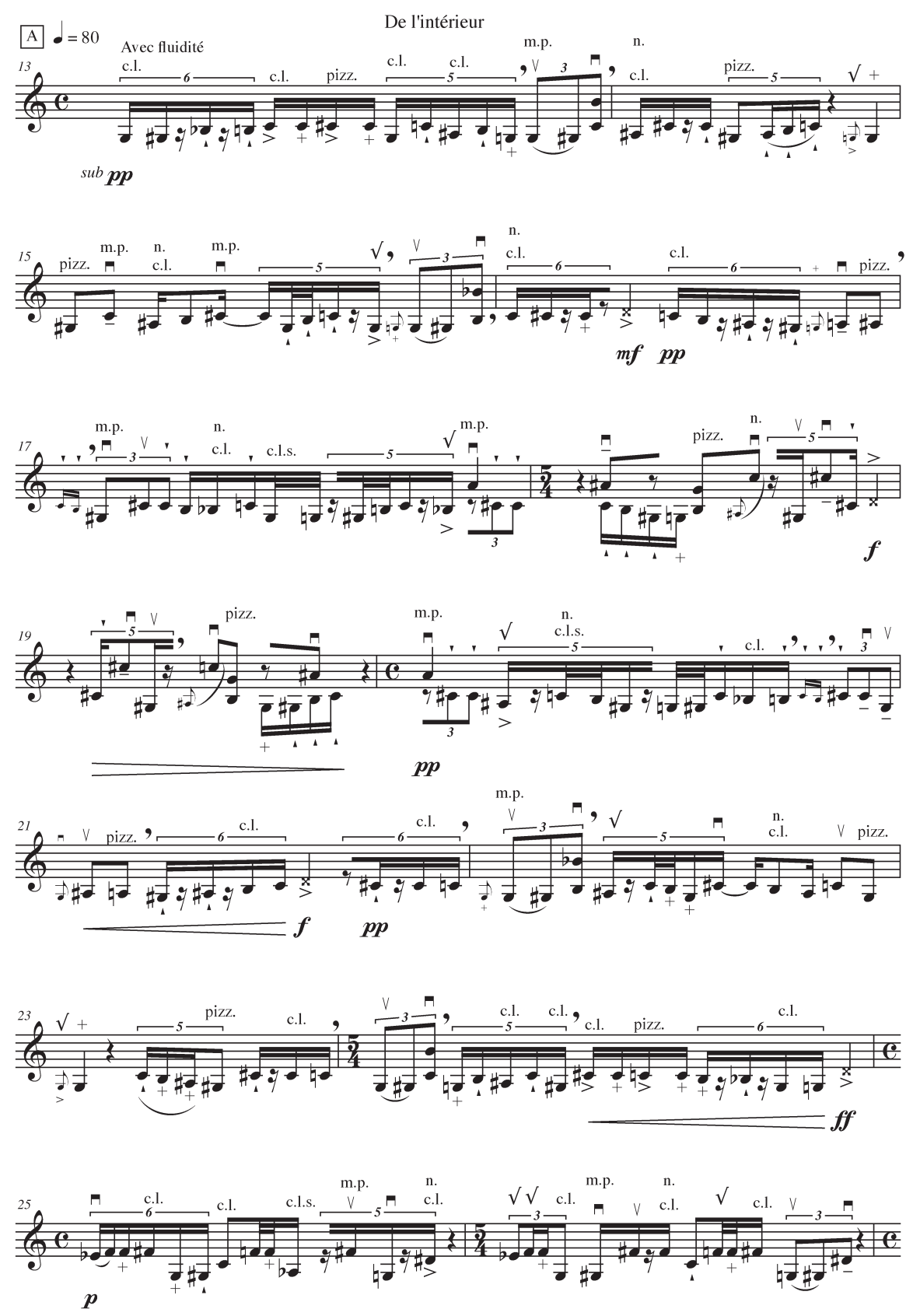

Création : 3 mars 2010, eXcentris, salle Cassavetes, Montréal.

Ensembles: Ensemble contemporain de Montréal (ECM+) et Proxima Centauri (France)/ Véronique Lacroix. 

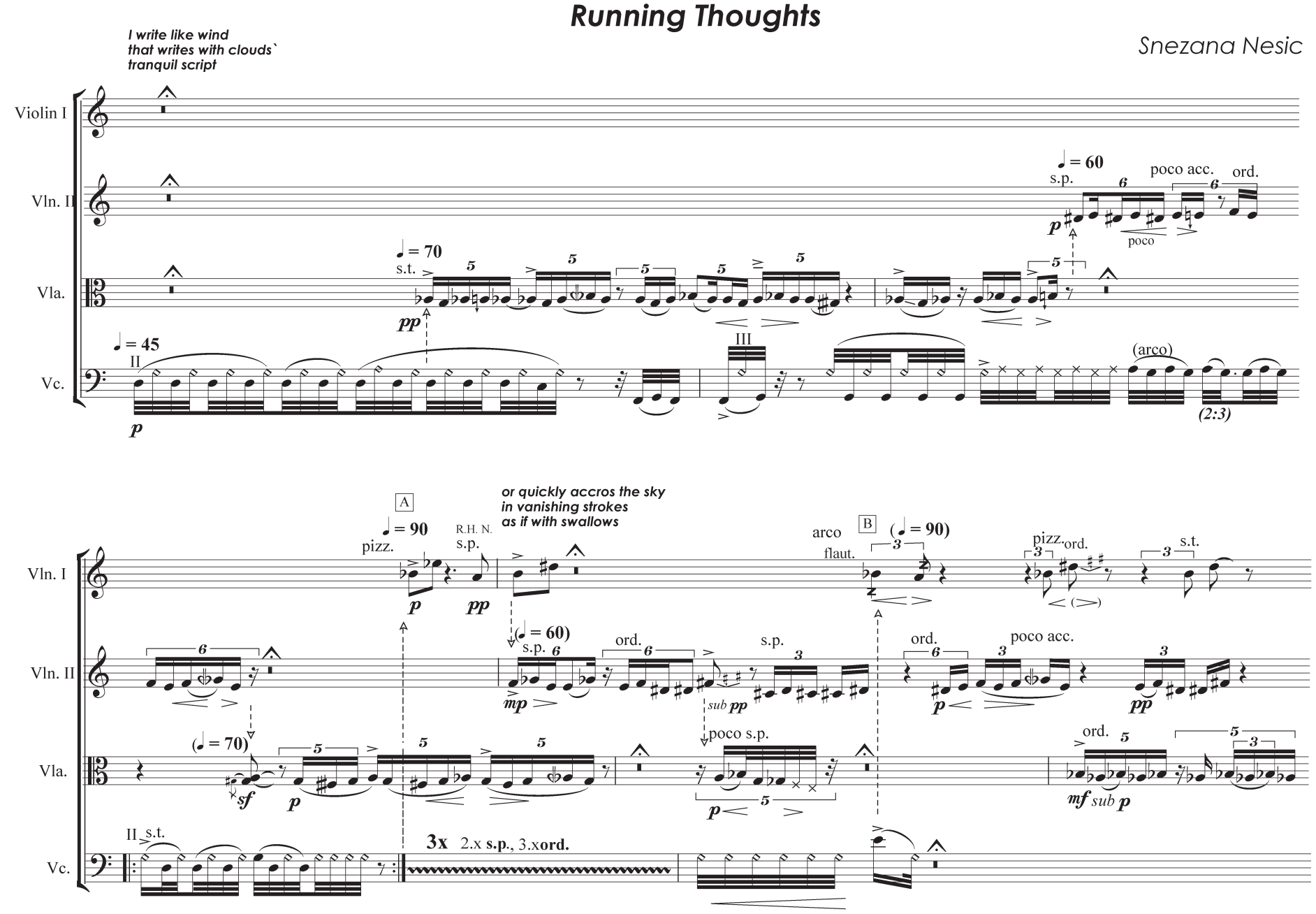

\section{Snezan Nesic Running Thoughts}



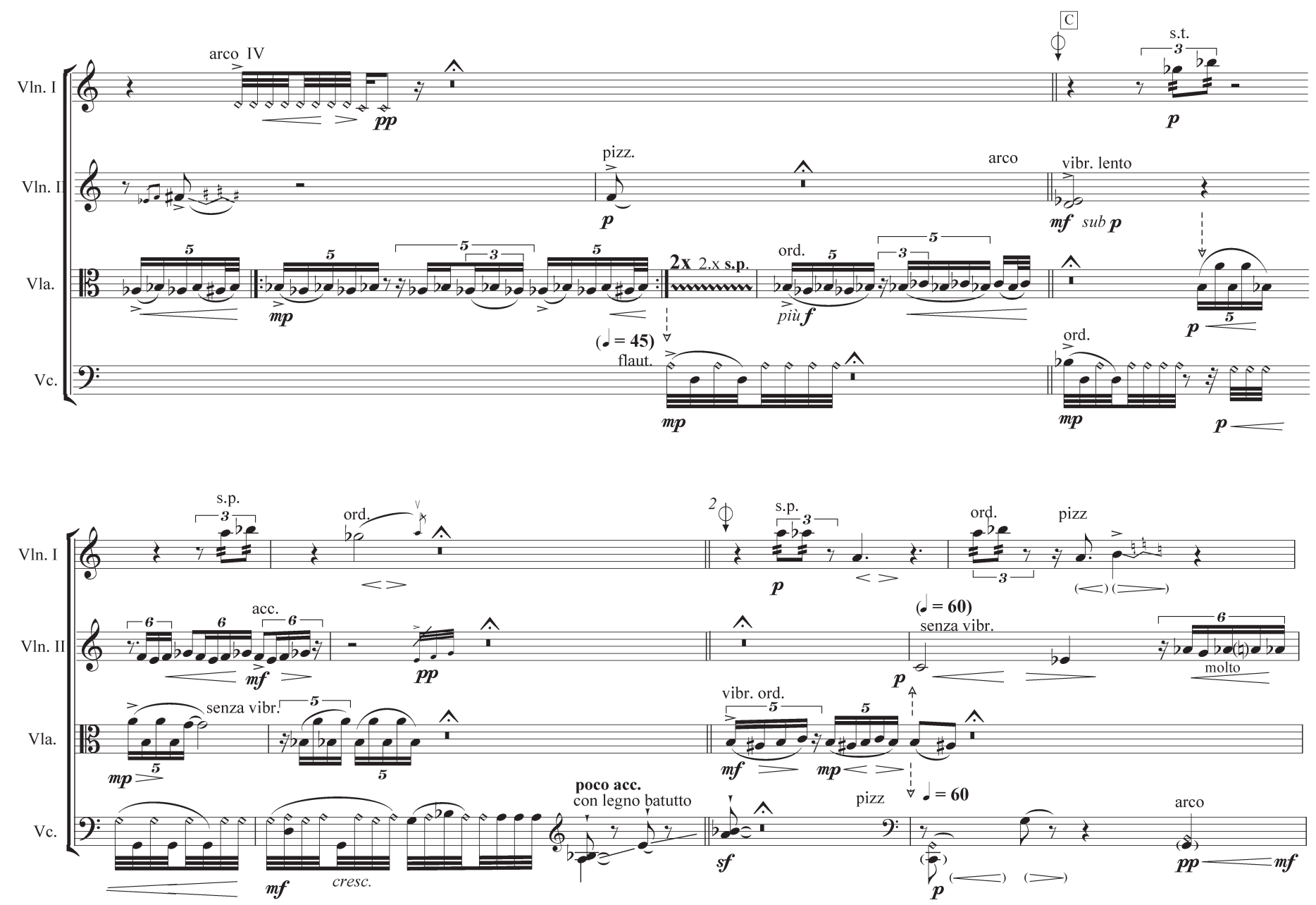

Premier prix du Quatrième Concours international de composition du Quatuor Molinari.

Création: 14 février 2010, Conservatoire de musique de Montréal.

Ensemble: Quatuor Molinari 

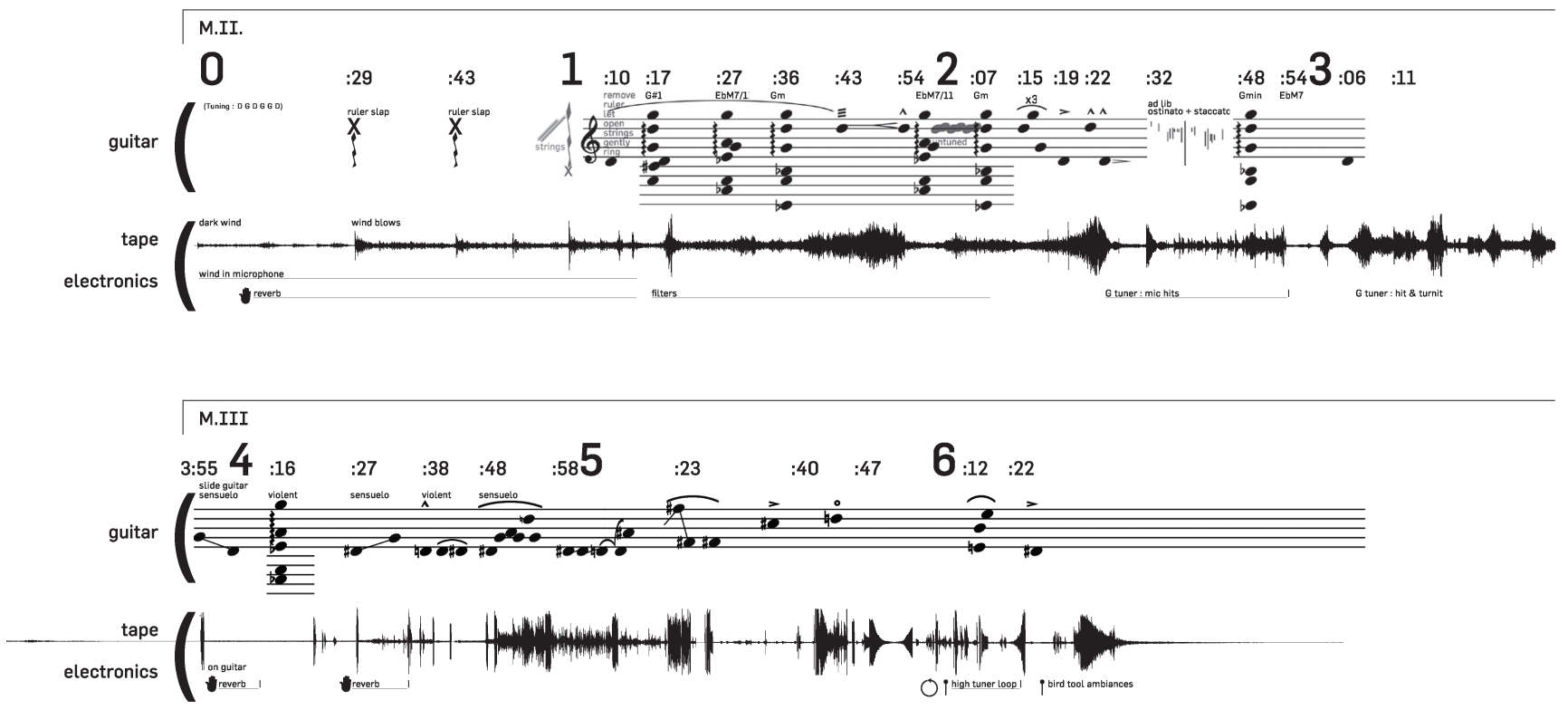

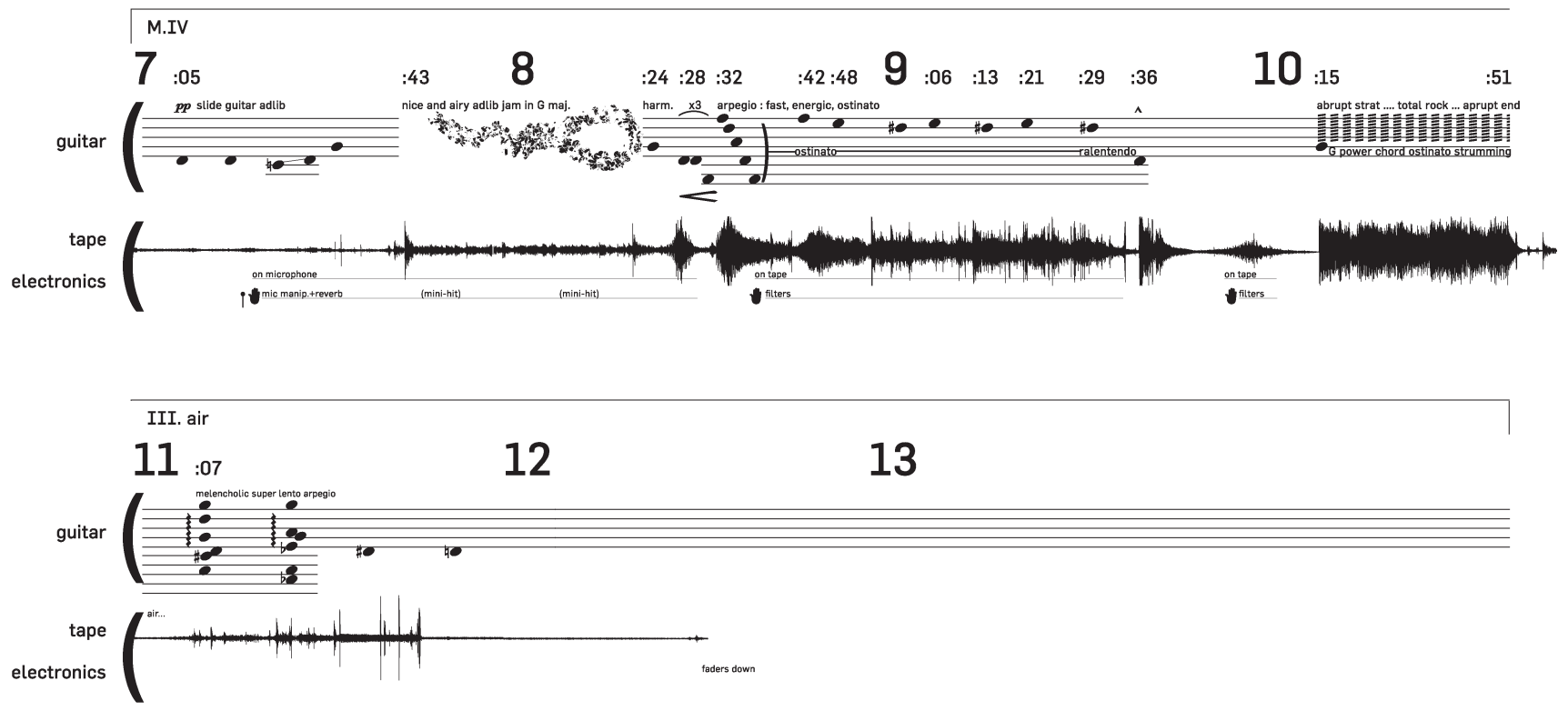

Pour guitare acoustique, bande 4 pistes et électronique en direct.

Création: 30 octobre 2010, durant Akousma 7, Monument-National, Studio Hydro-Québec, Montréal.

Guitariste: Simon Trottier 
2.«Thwack, Bang, Pow, Zap»

air de bravoure alternant les onomatopées du titre - en ralenti-bégayé et des vocalises virtuoses sur a-e-i-o-u-y; ce numéro suit une courte introduction instrumentale au tout début du spectacle, et est suivi par l'apparition des images de Mister Dégoût, et l'entrée en scène des trois personnages (début du texte dans le livret). Les hauteurs de notes sont libres lorsqu'il n'y a pas les 5 lignes habituelles, mais les registres approximatifs doivent être respectés.
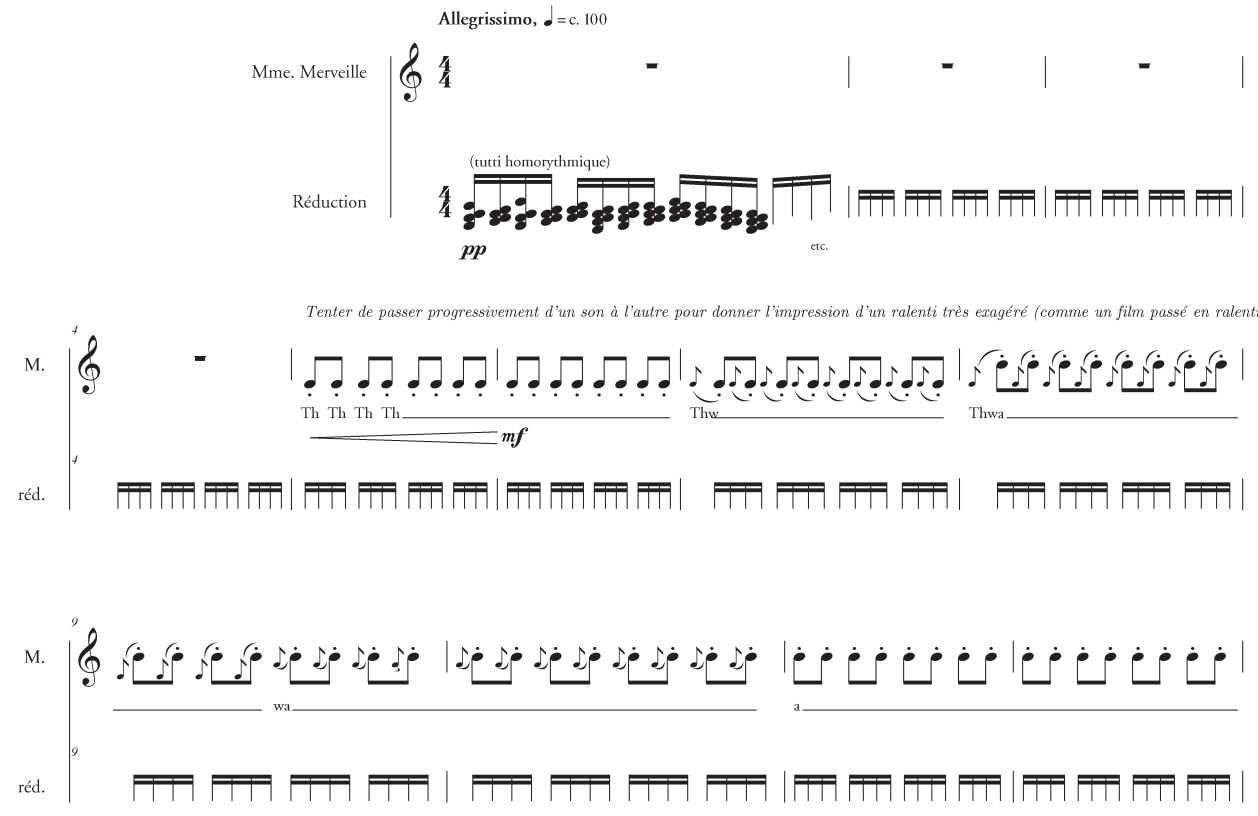

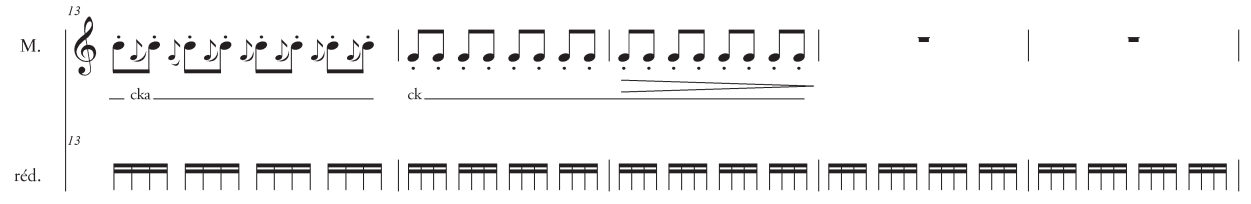
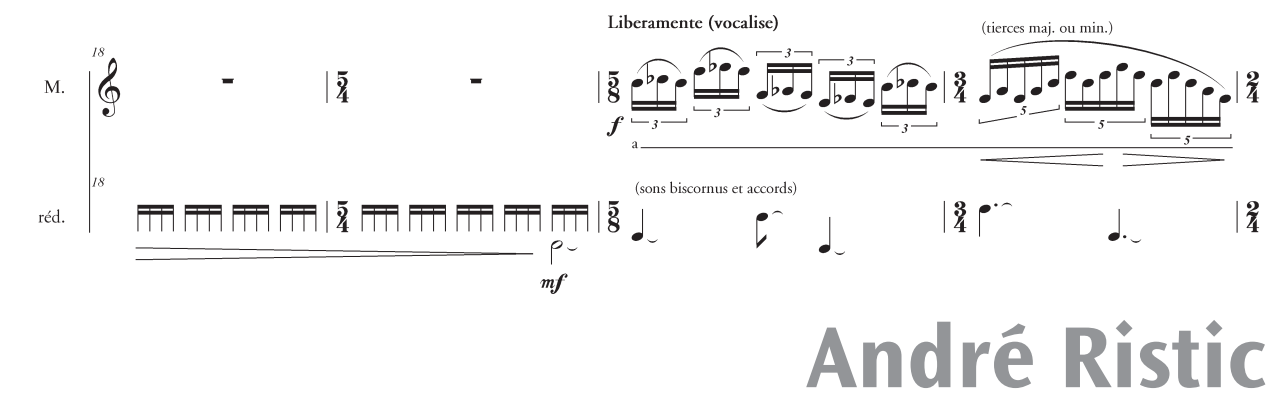

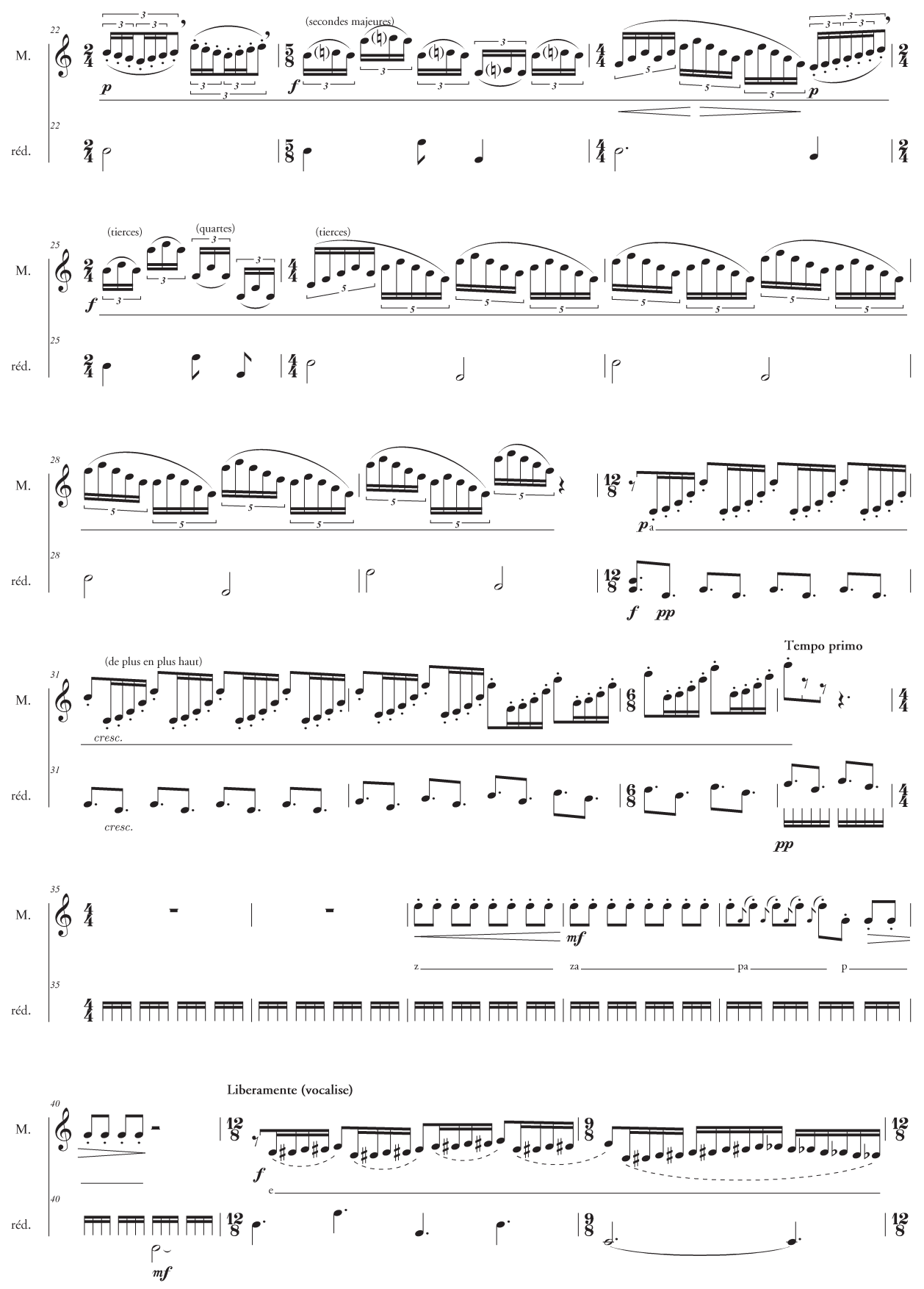

Opéra bédé.

Création : 6 mai 2010, Maison de la culture Frontenac, Montréal.

Ensemble: Ensemble contemporain de Montréal (ECM+)/Véronique Lacroix 

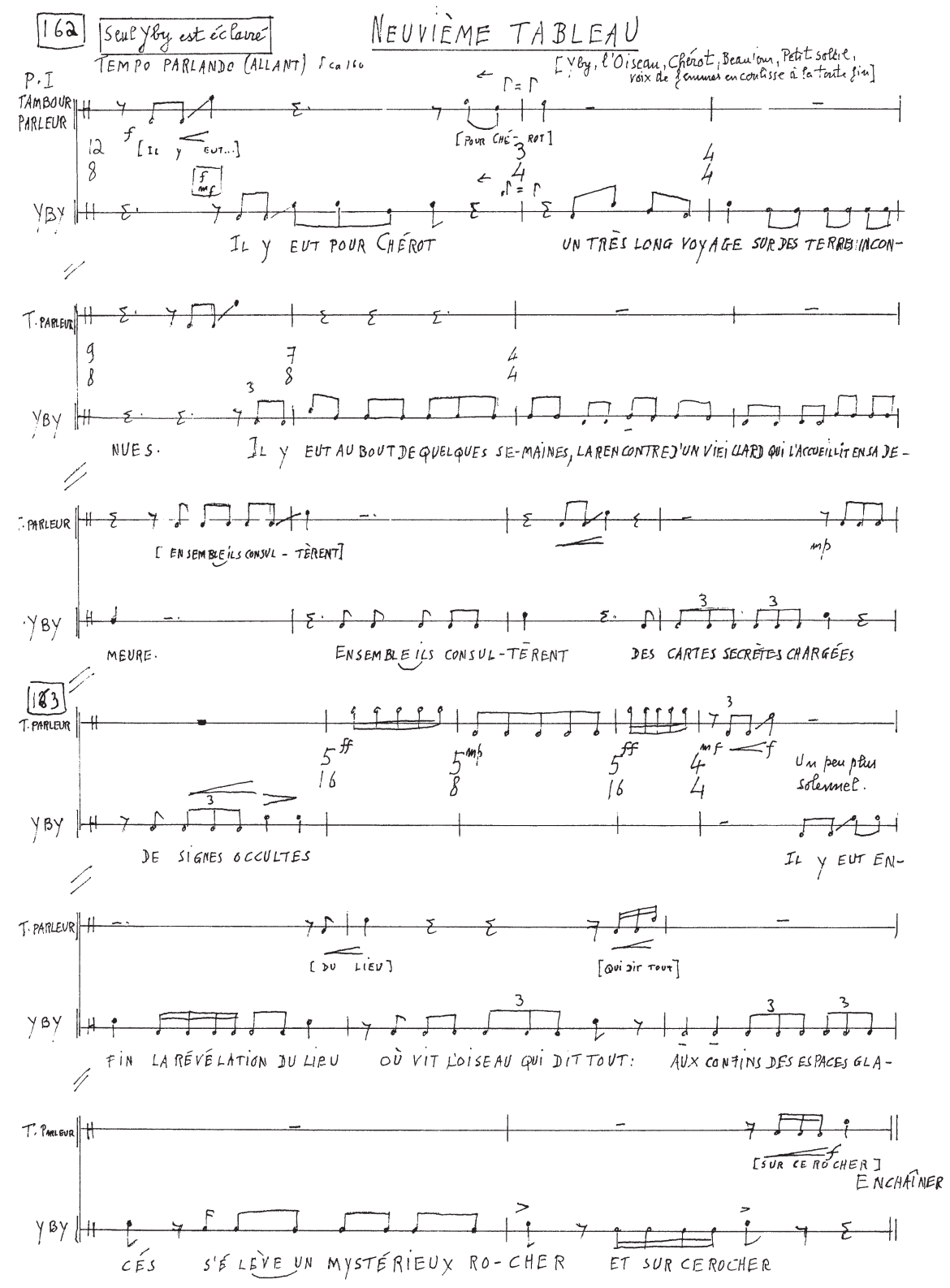
et l'oiseau qui dit la vérité 


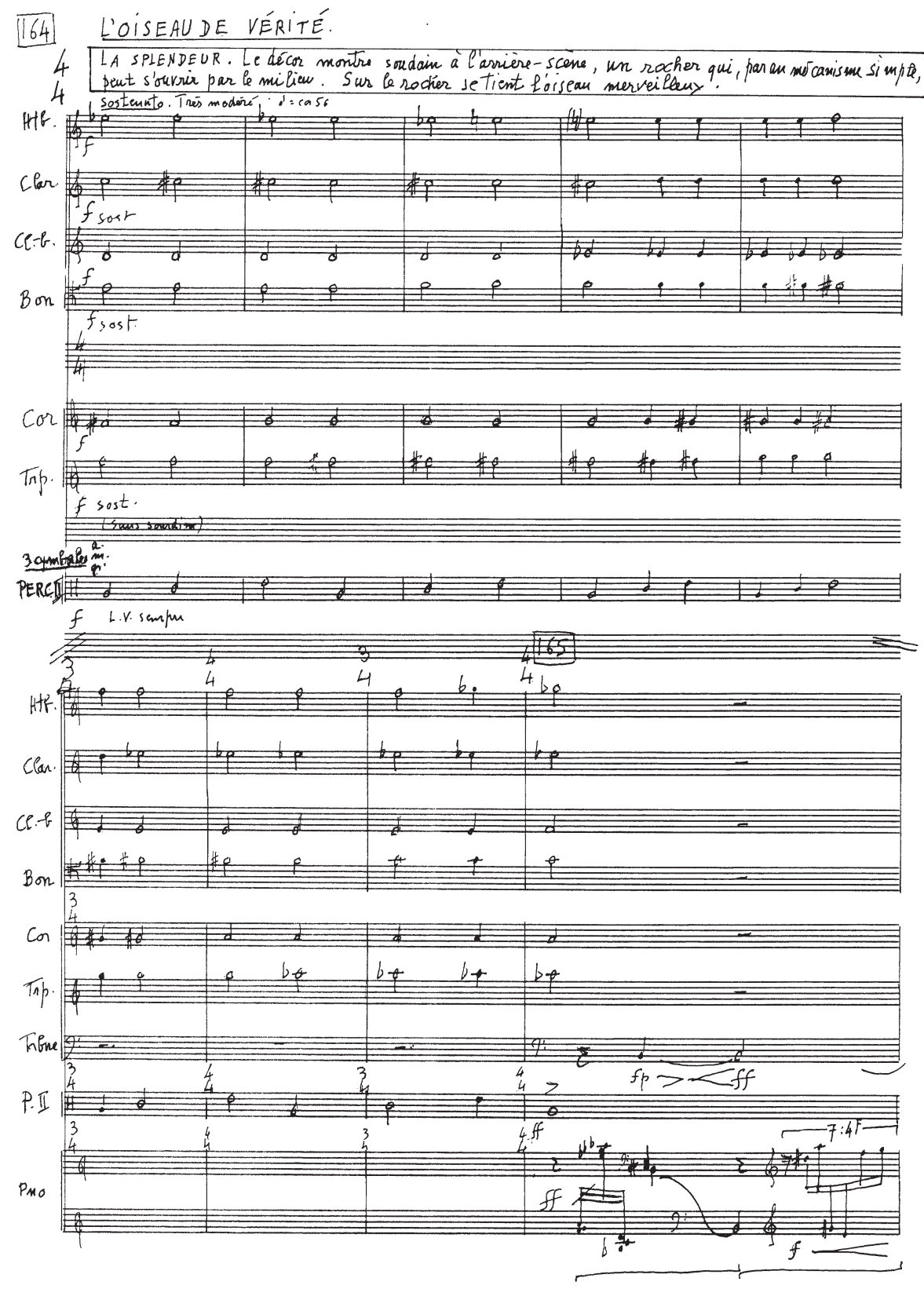

Opéra féerie.

Création: 19, 20 et 21 novembre 2009, Monument-National, salle Ludger-Duvernay, Montréal.

Ensemble: Nouvel Ensemble Moderne/Lorraine Vaillancourt

Production: Chants Libres 


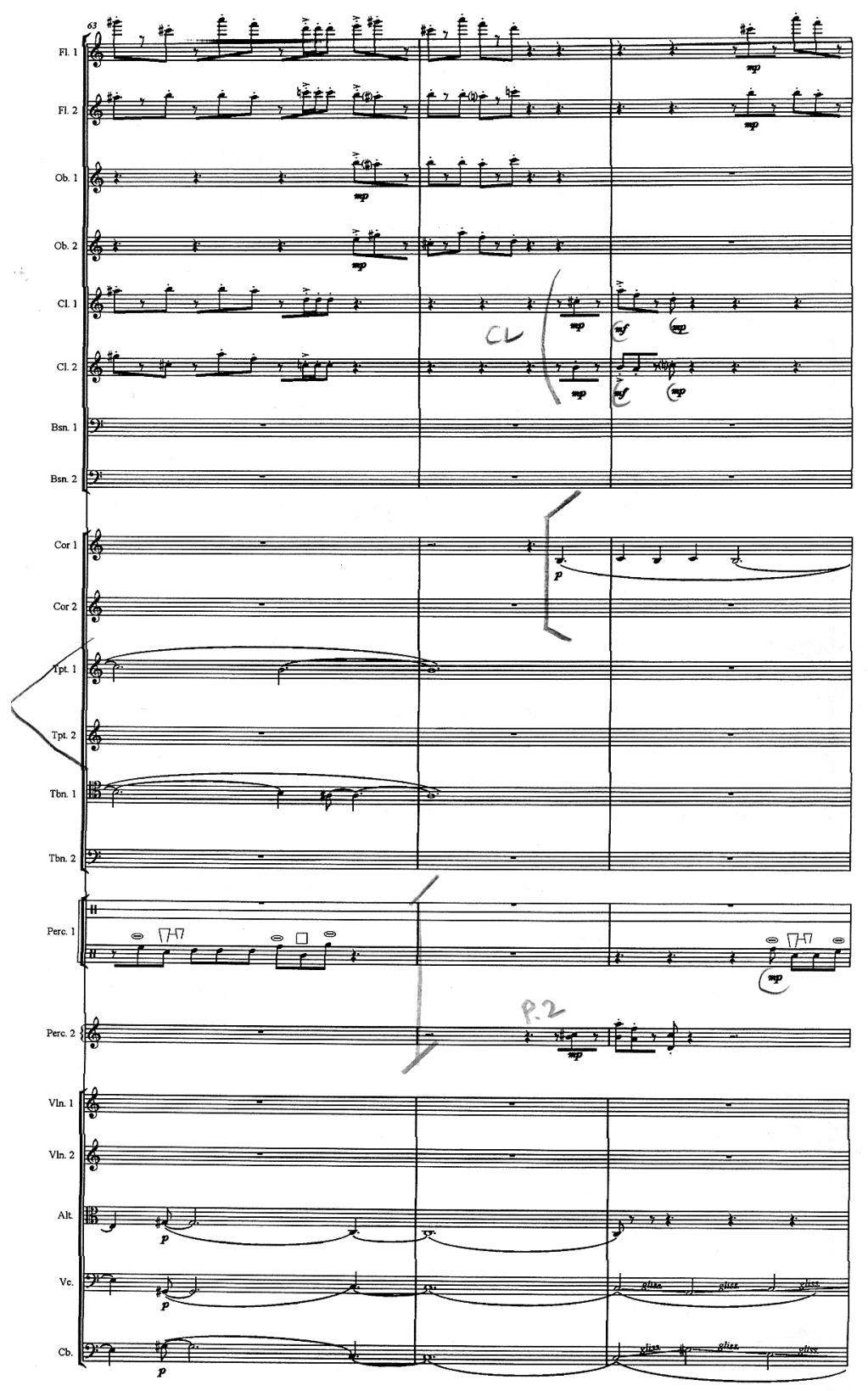

\section{René Orea-Sanchez Landocinogena}




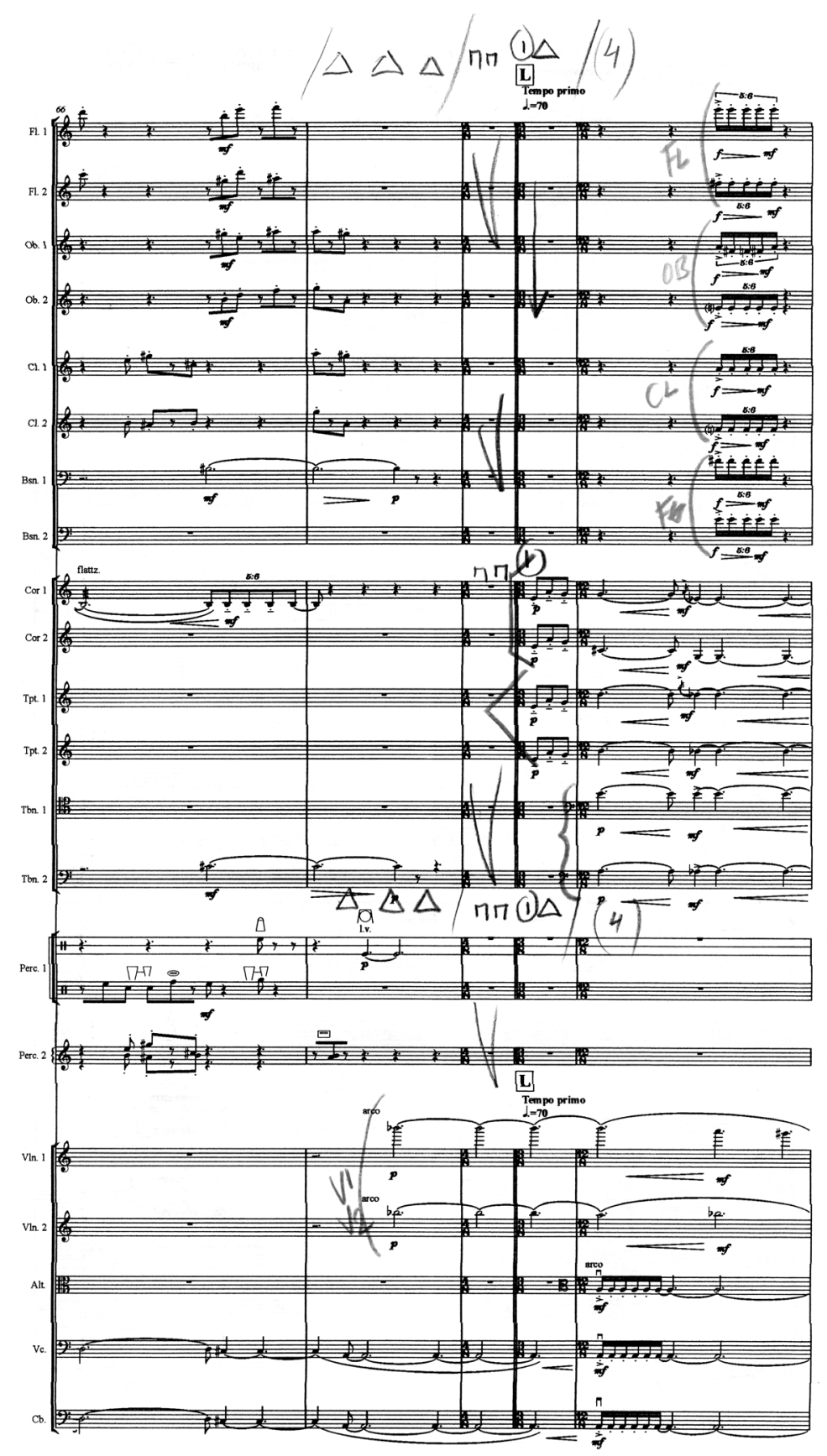

Création: 29 novembre 2009, Gesù, Centre de créativité, Montréal. 


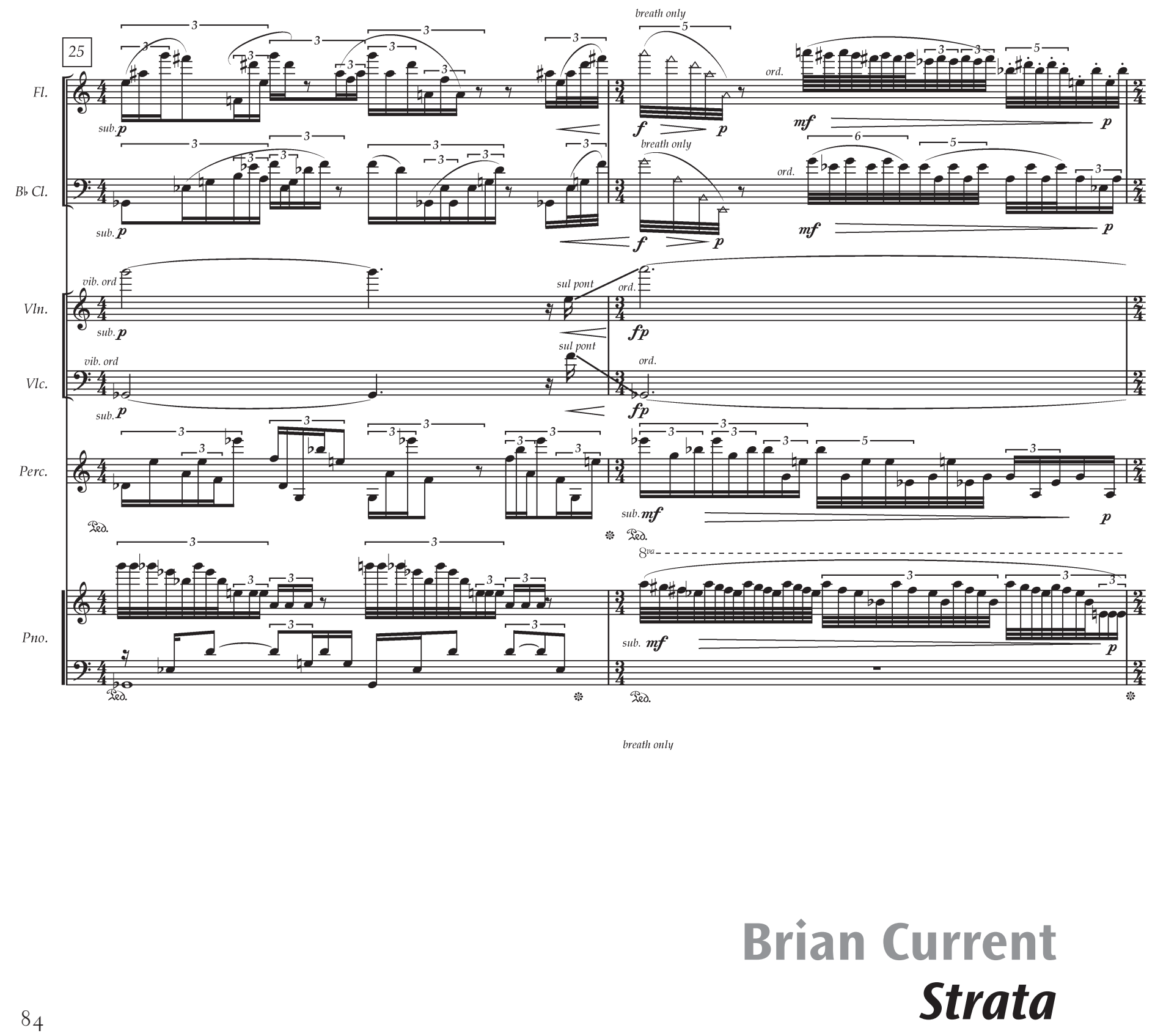




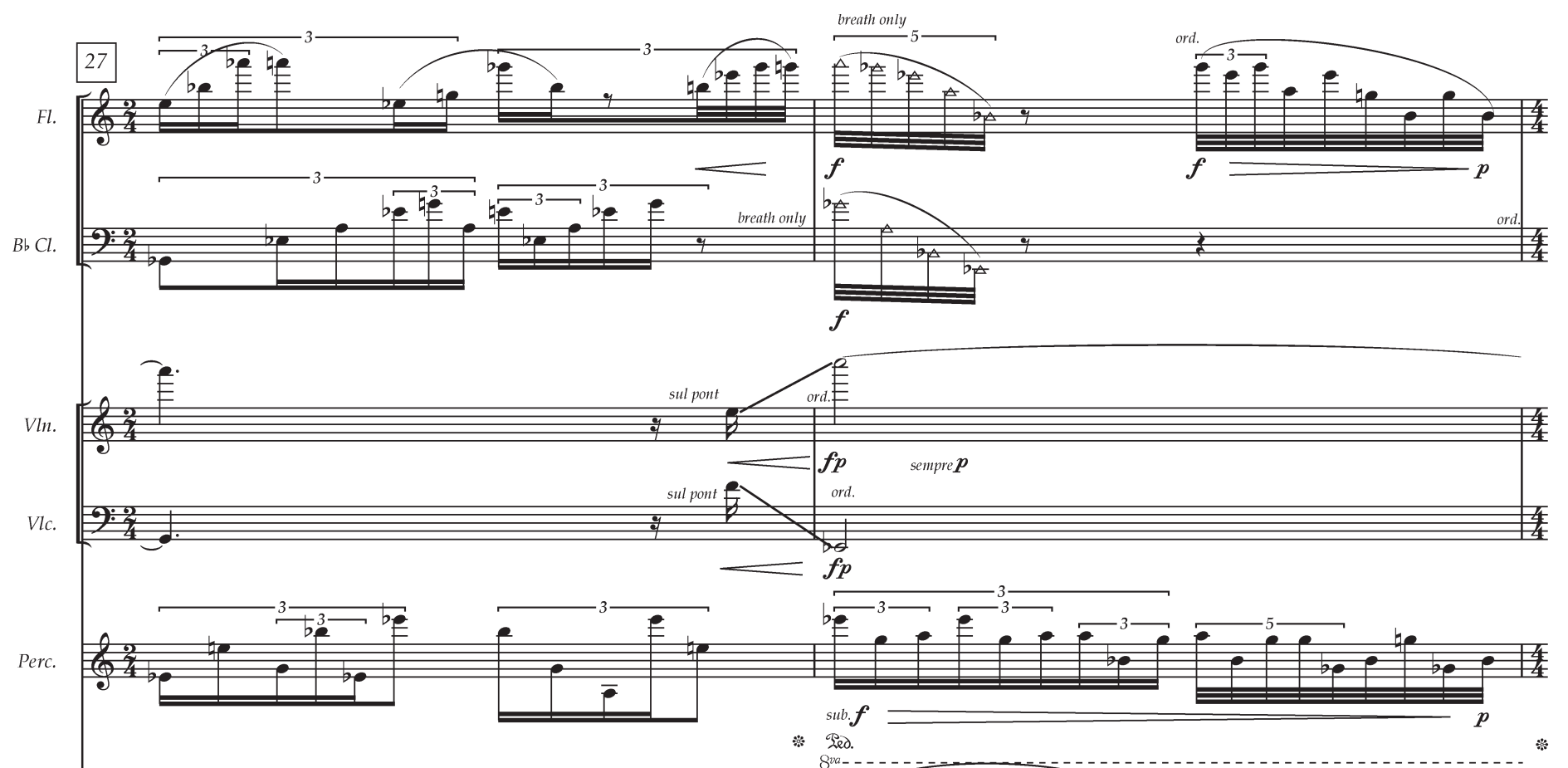

Tied

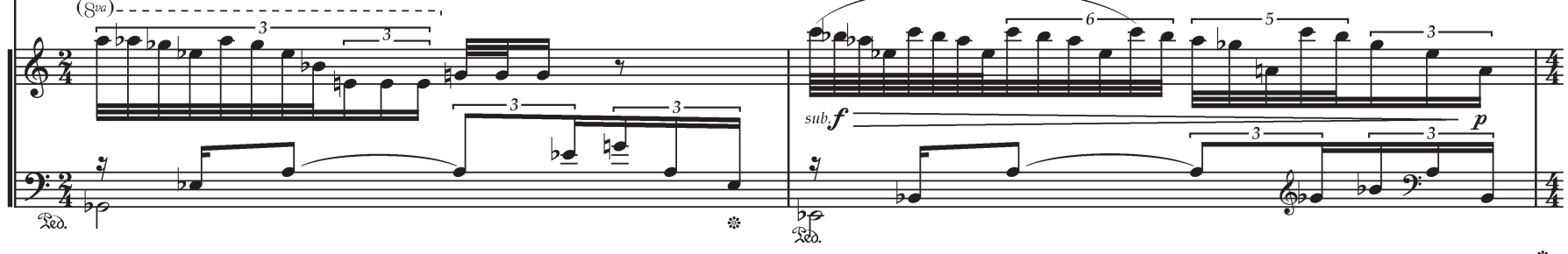

Création: 28 février 2011, San Francisco.

Ensemble: San Francisco Contemporary Music Players. 
Volées. Carillons d'oiseaux (pour Gilles Tremblay)
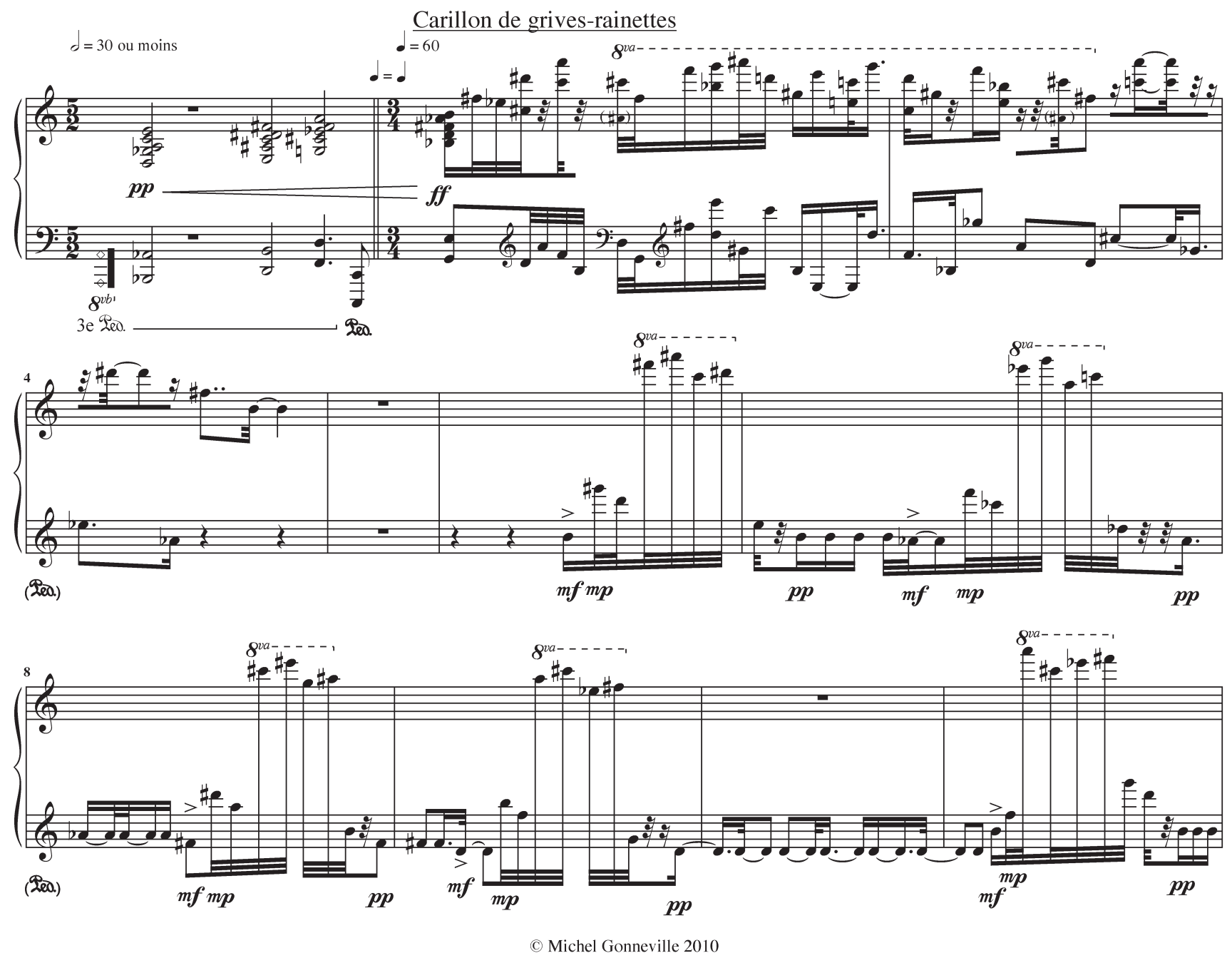

(C) Michel Gonneville 2010

\section{Michel Gonneville Volées. Carillons d'oiseaux (pour Gilles Tremblay)}




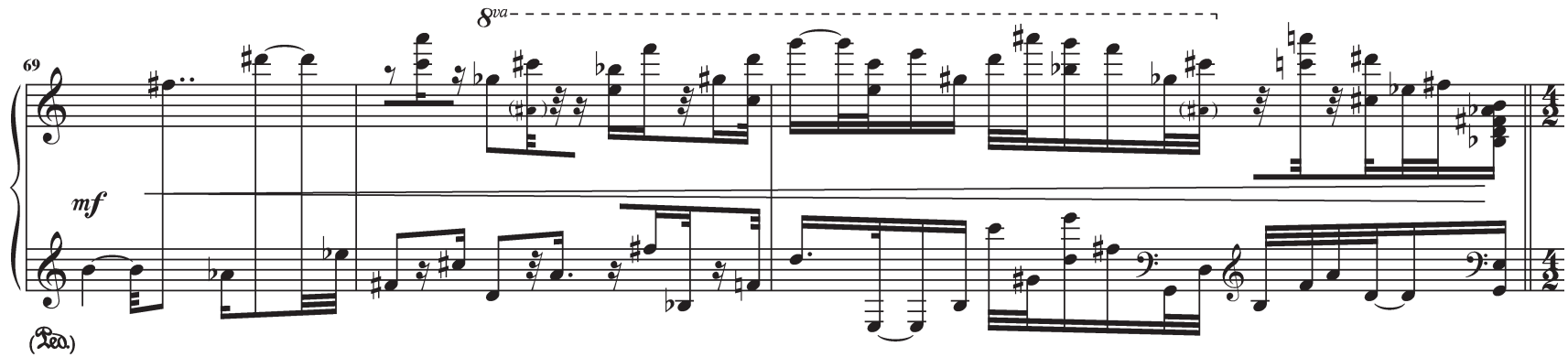

(200)

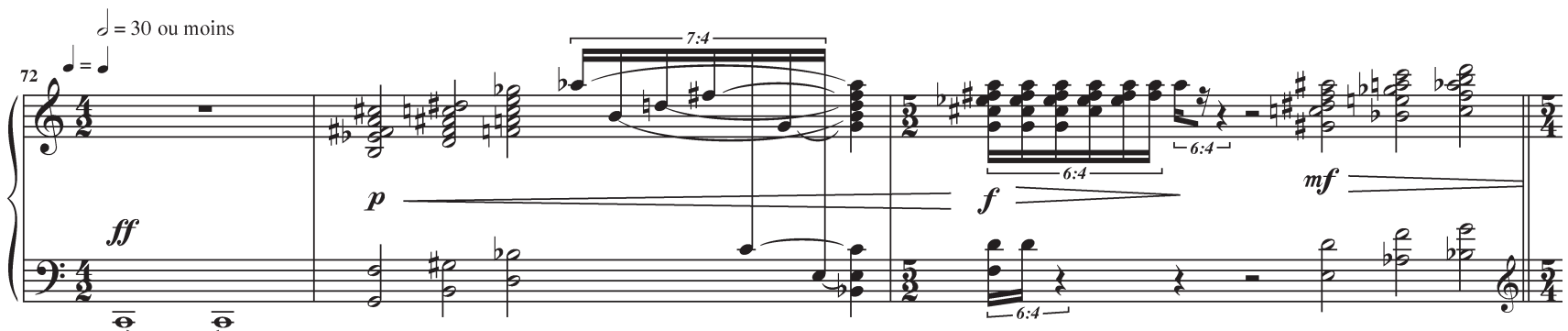
(200) $8^{\text {vb-n-n- }}$

Seo.

*

Résonances de pics-chamanes

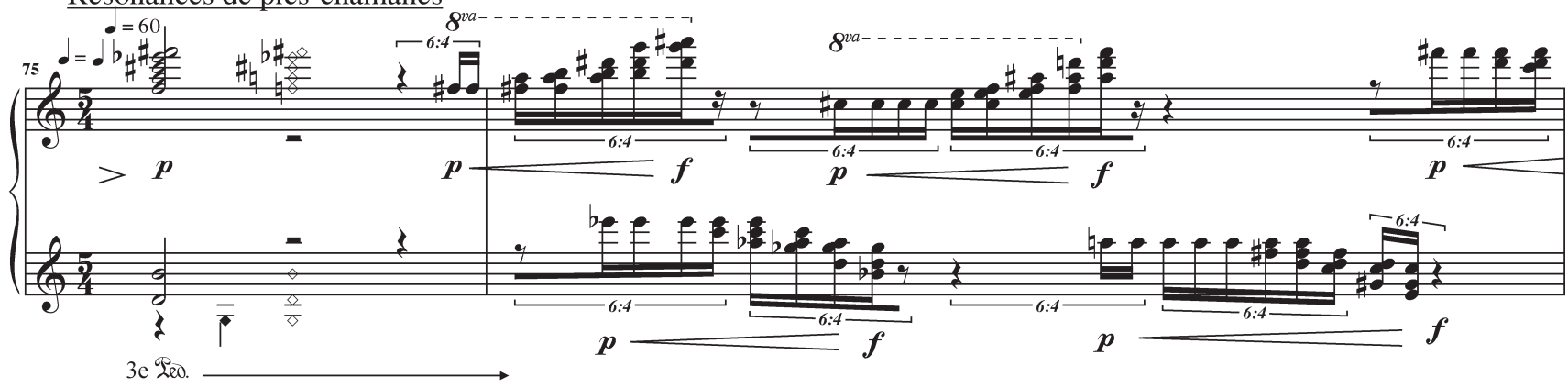

Création: 18 mars 2010, Salle Pierre-Mercure, Montréal.

Concert : Solstices (SMCQ)

Soliste: Louise Bessette 


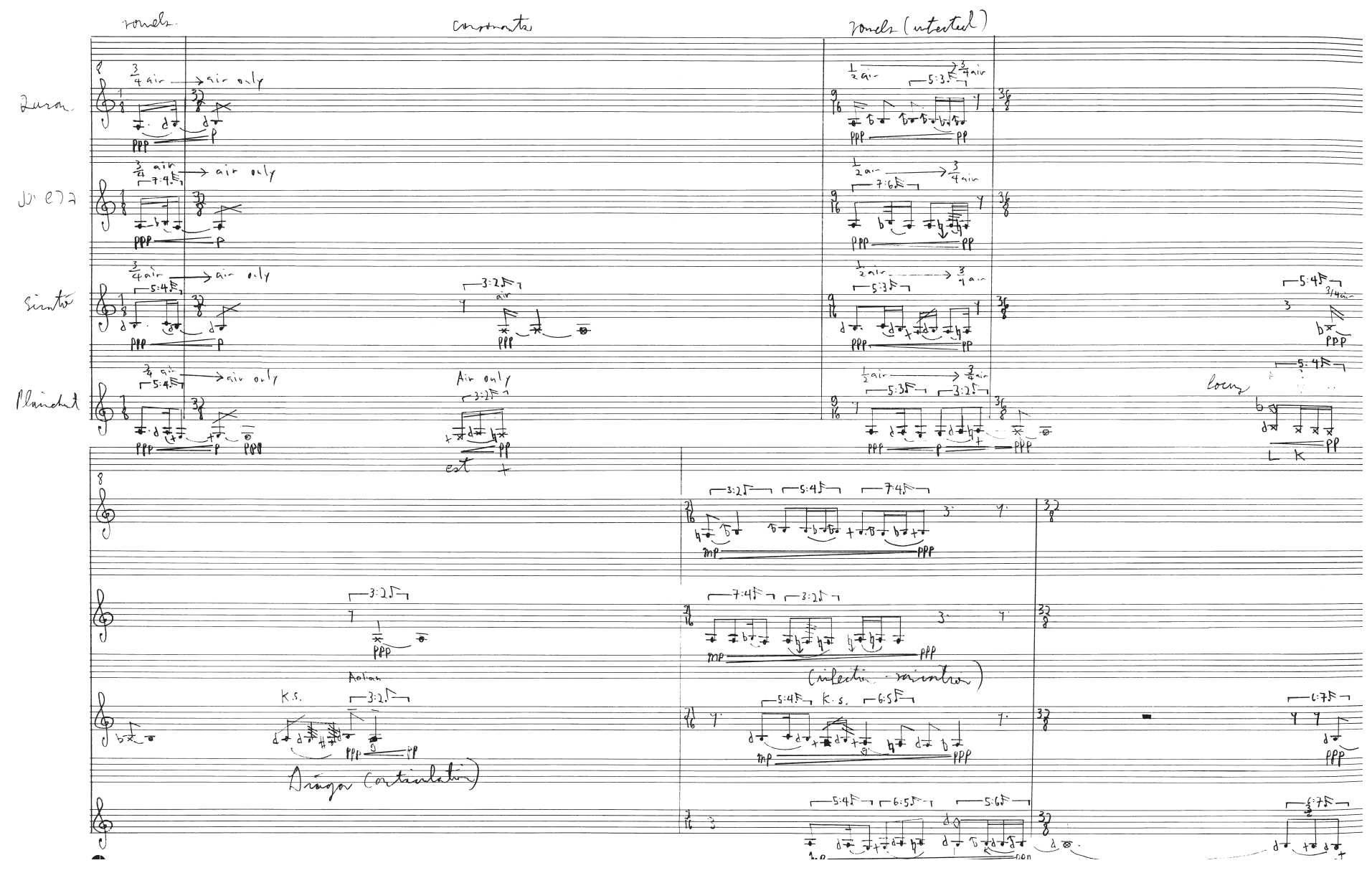

\section{Dániel Péter Bìró Udvarim Achadim}

Quatuor de saxophones et traitements électroniques. Création: 31 mars et $1^{\text {er }}$ avril 2010, Espace Dell'Arte, Montréal. 

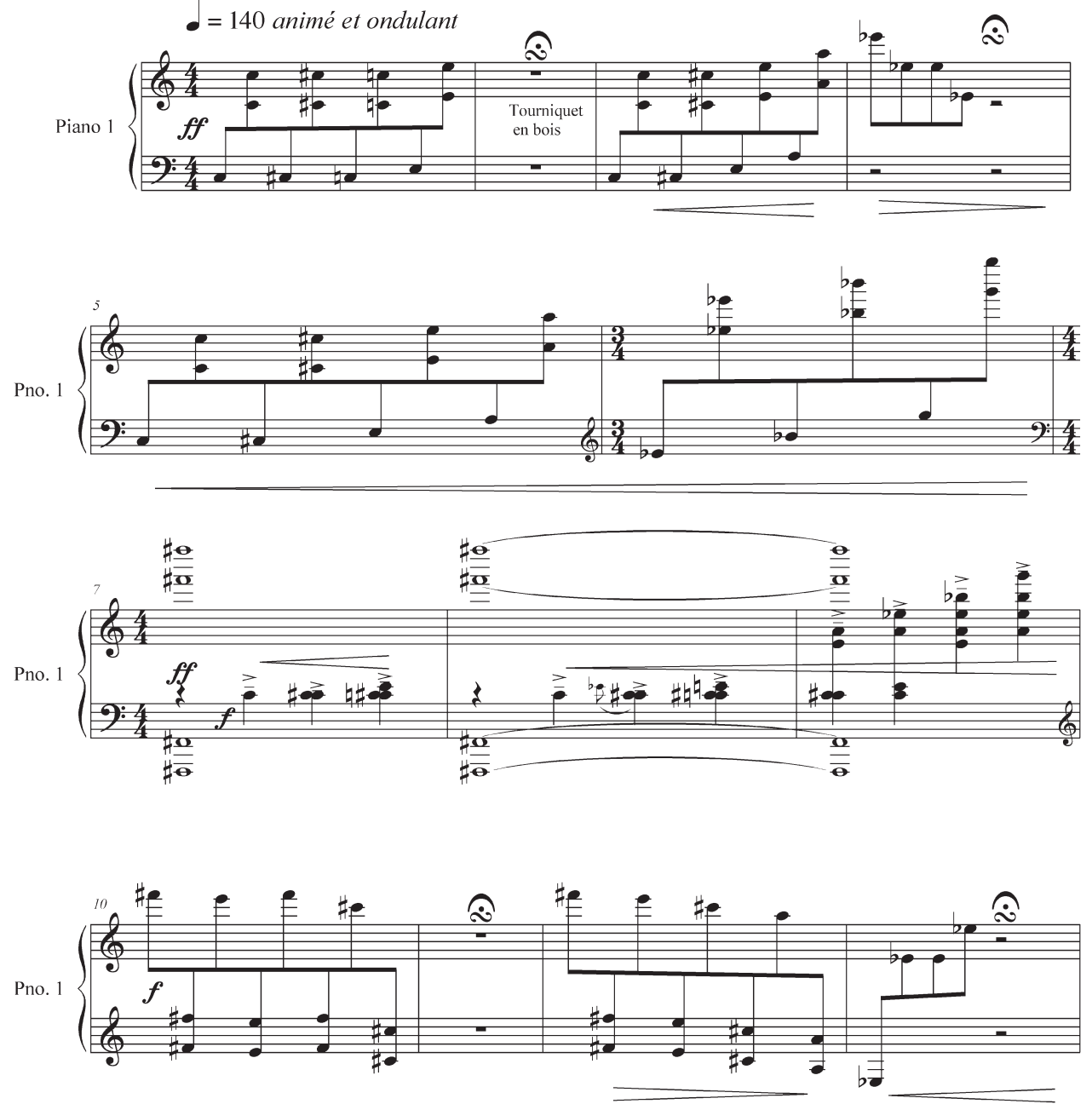

\section{Simon Bertrand}

22 miniatures pour piano, d'après les 22 arcanes majeures du Tarot de Marseille

Création : 8 mai 2010, Chapelle historique du Bon-Pasteur, Montréal. 


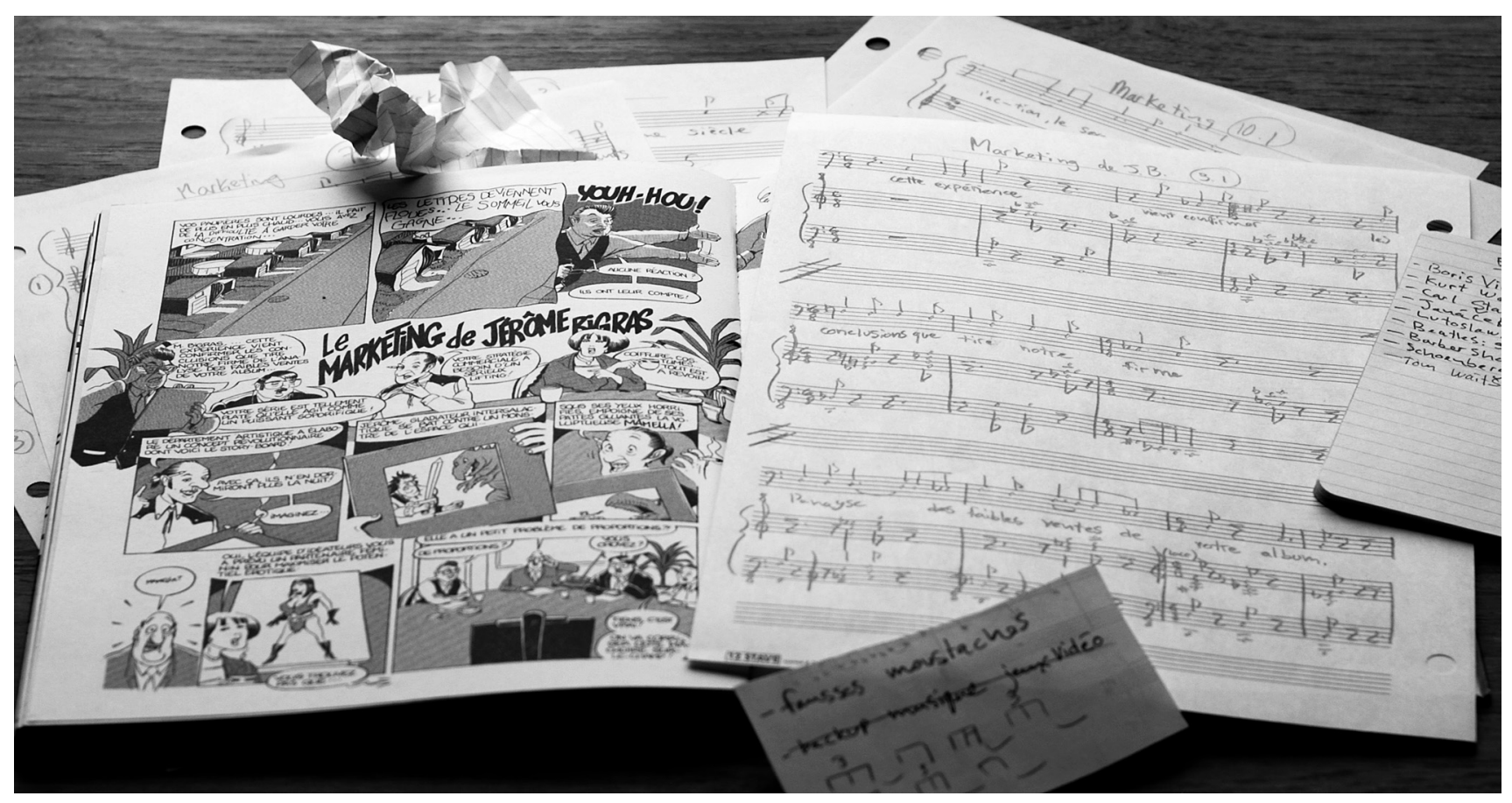

\section{Éric Champagne, Frédéric Chiasson, Benoît Côté, Maxime Goulet, Marielle Groven, Pierre Olivier Roy Bungalopolis}

Opéra cabaret autour des BD de Jean-Paul Eid Création : 20 mai 2010, Lion d'Or, Montréal. Ensemble: Prana 